Research in Astron. Astrophys. 2010 Vol.XX No.X, 000-000

http://www.raa-journal.org http://www.iop.org/journals/raa

Research in

Astronomy and

Astrophysics

\title{
Winding sense of galaxies around the Local Supercluster
}

\author{
B. Aryal ${ }^{1,2}$ \\ 1 Central Department of Physics, Tribhuvan University, Kirtipur, Nepal binil.aryal@uibk.ac.at \\ 2 Institut of Astro- and Particle Physics, Innsbruck University, Technikerstrasse 25, A-6020 \\ Innsbruck, Austria \\ Received [2010] [January] [14]; accepted [2010] [October] [23]
}

\begin{abstract}
We present an analysis of the winding sense (S and Z-shaped) of 1621 field galaxies that have radial velocity between $3000 \mathrm{~km} \mathrm{~s}^{-1}$ to $5000 \mathrm{~km} \mathrm{~s}^{-1}$. The preferred alignments of S- and Z-shaped galaxies are studied using chi-square, autocorrelation and the Fourier tests. We classified total galaxies into 32 subsamples and noticed a good agreement between the position angle (PA) distribution of S- and Z-shaped galaxies. The homogeneous distribution of the S- and Z-shaped galaxies is noticed for the late-type spirals ( $\mathrm{Sc}, \mathrm{Scd}, \mathrm{Sd}$ and $\mathrm{Sm}$ ) than that of the early-types ( $\mathrm{Sa}, \mathrm{Sab}, \mathrm{Sb}$ and $\mathrm{Sbc}$ ). A significant dominance of S-mode galaxies is noticed in the barred spirals. A random alignment is noticed in the PA-distribution of Z- and S-mode spirals. In addition, homogeneous distribution of the S- and Z-shaped galaxies is found to be invariant under the global expansion. The PAdistribution of the total S-mode galaxies is found to be random, whereas preferred alignment is noticed for the total Z-mode galaxies. It is found that the galactic planes of Z-mode galaxies tend to lie in the equatorial plane.
\end{abstract}

Key words: spiral galaxies - clusters: individual (Local Supercluster)

\section{INTRODUCTION}

Differential rotation in a galaxy's disc generate density waves in the disc, leading to spiral arms. According to gravitational theory, the spiral arms born as leading and subsequently transform to trailing modes. With the passage of time, the spiral pattern deteriorates gradually by the differential rotation of the equatorial plane of the galaxy, but the bar structure persists for a long time (Oort 1970a). This structure can again regenerate spiral pattern in the outer region. Thus, a close relation between the origin of the arms in the spirals and barred spirals can not be denied (Oort 1970b).

Land et al. (2008) studied the distribution of projected spin vectors of $\sim 37000$ spiral galaxies taken from Solon Dizital Sky Survey. They did not notice any evidence for overall preferred handedness of Universe. In a similar study, Longo (2007) found evidence for a preferred axis. Sugai \& Iye (1995) used statistics and studied the winding sense of galaxies (S- and Zpatterns) in 9825 spirals. No significant dominance from a random distribution is noticed. Aryal \& Saurer (2005) studied the spatial orientations of spin vectors of 4073 galaxies in the Local Supercluster. No preferred alignment is noticed for the total sample. These results hint that the distribution of angular momentum of galaxies is entirely random in two- (S- and Z-shaped) and three-dimensional (spin vector) analysis provided the database is rich. 
In order to understand true structural modes (leading or trailing) of spiral galaxies, we need to know the direction of the spiral pattern ( $\mathrm{S}$ - or Z-patterns), the approaching and receding sides and the near and far parts, since galaxies are commonly inclined in space to the line of the sight. The S and Z-patterns can be determined from the image of the galaxy. Similarly, the approaching and receding sides can be defined if spectroscopy data on rotation is available. The third one is fairly hard to established. For this, Pasha (1985) used 'tilt' criteria and studied the sense of winding of the arms in 132 spirals. He found 107 spirals to have trailing arm. Thomasson et al. (1989) studied theoretically and performed $N$-body simulations in order to understand the formation of spiral structures in retrograde galaxy encounters. Interestingly, they noticed the importance of halo mass. They concluded that the spirals having halos with masses larger than the disk mass exhibit leading pattern. Thus, the makeup of galactic haloes is important to cosmology in order to understand the evolution of galaxies.

By considering the group of transformations acting on the configuration space, Capozziello \& Lattanzi (2006) predicted that the progressive loss of inhomogeneity in the S- and Z-shaped galaxies might have some connection with the rotationally-supported (spirals, barred spirals) and randomized stellar systems (lenticulars, ellipticals).

The preferred alignments of galaxies can be an indicator of initial conditions when galaxies and clusters formed provided the angular momenta of galaxies have not been altered too much since their formation. A useful property of galaxies in clusters for which theories make different predictions is the angular momentum distribution. The 'Pancake model' by Doroshkevich (1973), the 'Hierarchy model' by Peebles (1969) and, the 'Primordial vorticity model' by Ozernoy (1978) predict different scenarios concerning the formation of large-scale structure. Thus, the study of galaxy orientation has the potential to yield important information regarding the formation and evolution of cosmic structures. In this work, we present an analysis of winding sense and preferred alignments of galaxies that have radial velocity (RV) $3000 \mathrm{~km}$ $\mathrm{s}^{-1}$ to $5000 \mathrm{~km} \mathrm{~s}^{-1}$. These are field galaxies. We intend to study the importance of winding sense in order to understand the true structural modes (i.e., leading and trailing arm) of the galaxy. We expect to study the following: (1) Are the distribution of S- and Z-shaped galaxies homogeneous in the field? (2) Is there any correlation between the preferred alignment and the winding sense of galaxies? (3) Does radial velocity dependence exist concerning winding sense of galaxies? and finally, (4) What can we say about the distribution of true structural modes (i.e., leading or trailing arm) of galaxies in the large scale structure?

This paper is organized as follows: in Sect. 2 we describe the method of data reduction. In Sect. 3 we give a brief account of the methods and the statistics used. Finally, a discussion of the statistical results and the conclusions are presented in Sects. 4 and 5.

\section{THE SAMPLE: DATA REDUCTION}

Eighteen catalogues were used for the data compilation. A list of the catalogues and their references are given in Table 1. The abbreviations given in the first column of Table 1 are as follows: NGC - New General Catalogue, UGC - Uppsala General Catalogue of Galaxies, ESO - ESO/Uppsala Survey of the ESO (B) Atlas, IC - Index Catalogue, MCG - Morphological Galaxy Catalogue, UGCA - Uppsala obs. General Catalogue, Addendum, CGCG - Catalogue of Galaxies and Clusters of Galaxies, KUG - Kiso Ultraviolet Galaxy Catalogue, MRK Markarian Galaxy Catalogue, MESSIER - Catalogue des nebuleuses et des amas d'etoiles, BCG - Brandner+Grebel+Chu Catalogue, LSBG - Low Surface Brightness Galaxies, SBS - Second Byurakan Survey, LCRS - Las Companas Red Shift Survey, DDO - David Dunlap Observatory Publications, IRAS - Infrared Astronomical Satellite, SGC - Southern Galaxy Catalogue and UM - University of Michigan: Curtis Schmidt-thin prism survey for extragalactic emission-line objects: List I-V.

The NASA/IPAC extragalactic database (NED, http://nedwww.ipac.caltech.edu/) was used to compile these catalogues. The main editing process was as follows: first, galaxies having 


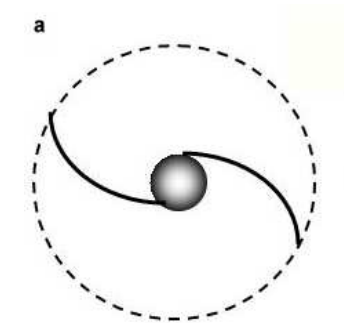

S-mode

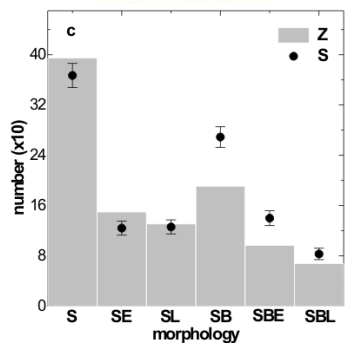

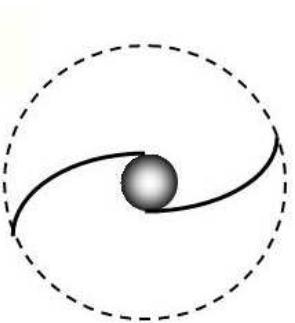

Z-mode

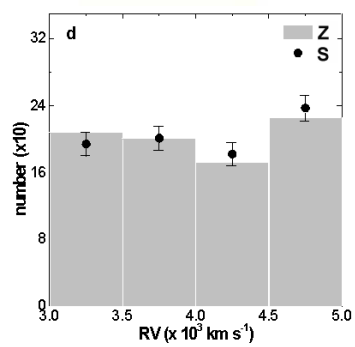

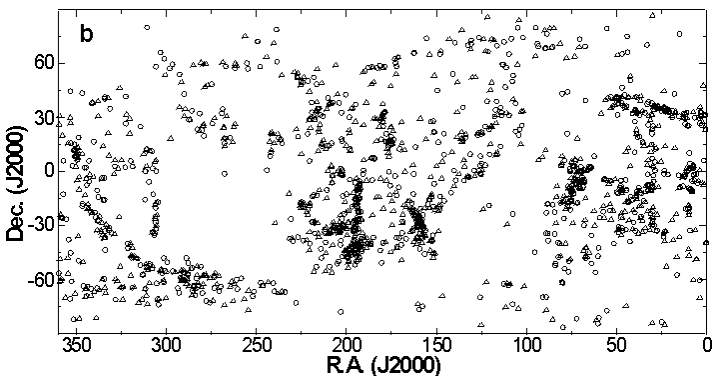
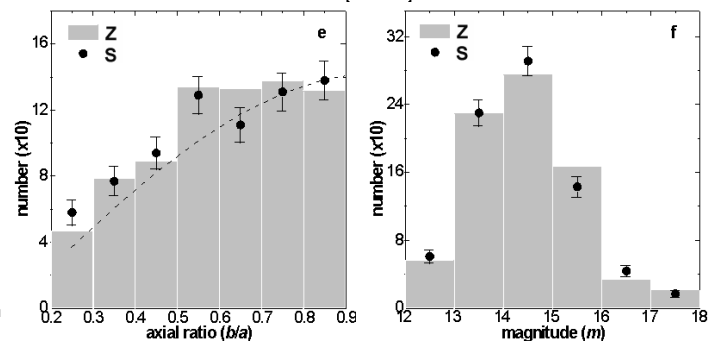

Fig. 1 (a) A sketch representing the winding sense ( $\mathrm{S}$ or $\mathrm{Z}$ ) of the galaxy. (b) Allsky distribution of Z-mode $(\triangle)$ and S-mode (o) galaxies that have RVs in the range $3000 \mathrm{~km} \mathrm{~s}^{-1}$ to $5000 \mathrm{~km} \mathrm{~s}^{-1}$. The morphology (c), radial velocity (d), axial ratio (e) and the magnitude (f) distribution of $\mathrm{Z}$ and $\mathrm{S}$-mode galaxies in our database. The statistical $\pm 1 \sigma$ error bars are shown for the S-mode $(\bullet)$ subsample. The dashed line (e) represents the expected distribution.

RVs in the range $3000 \mathrm{~km} \mathrm{~s}^{-1}$ to $5000 \mathrm{~km} \mathrm{~s}^{-1}$ were collected. We downloaded the image of all these galaxies from NED in FITS format. The second step was to compile the morphology of these galaxies from the catalog. A galaxy with doubtful morphology (eg., 'S?', 'S0' or 'Sa') is omitted. Finally, the position angles (PAs) of galaxies were added from the UGC, ESO, and Third Reference Catalogue of Bright Galaxies (de Vaucouleurs et al. 1991).

There were two clusters Abell $0426\left(\alpha(\mathrm{J} 2000)=03^{\mathrm{h}} 18^{\mathrm{m}} 36.4^{\mathrm{s}}, \delta(\mathrm{J} 2000)=+41^{\circ} 30^{\prime} 54^{\prime \prime}\right)$ and Abell $3627\left(\alpha(\mathrm{J} 2000)=16^{\mathrm{h}} 15^{\mathrm{m}} 32.8^{\mathrm{s}}, \delta(\mathrm{J} 2000)=-60^{\circ} 54^{\prime} 30^{\prime \prime}\right)$ in our region. These clusters have mean RVs $5366 \mathrm{~km} \mathrm{~s}^{-1}(75 \pm 5 \mathrm{Mpc})$ and $4881 \mathrm{~km} \mathrm{~s}^{-1}$ (63 $\pm 4 \mathrm{Mpc}$ ), respectively (Abell, Corwin \& Olowin 1989, Struble \& Rodd 1999). We removed the galaxies belong to the cluster Abell 0426 using the catalog established by Brunzendorf \& Meusinger (1999). For the cluster Abell 3627 galaxies, we used Photometric Atlas of Northern Bright Galaxies (Kodaira, Okamura \& Ichikawa 1990) and Uppsala Galaxy Catalogue (Nilson 1973). There were 174 galaxies belongs to these clusters in our database. We remove these galaxies.

The RVs were compiled from Las Campanas Redshift Survey (Shectman 1996). The PAs and the diameters of galaxies were added from the Uppsala Galaxy Catalogue (Nilson 1973), Uppsala obs. General Catalogue, Addendum (Nilson 1974), Photometric Atlas of Northern Bright Galaxies (Kodaira, Okamura, \& Ichikawa 1990), ESO/Uppsala Survey of the European Southern Observatory (Lauberts 1982), Southern Galaxy Catalogue (Corwin et al. 1985) and Third Reference Catalogue of Bright Galaxies (de Vaucouleurs et al. 1991).

In the NED, 6493 galaxies having RVs $3000 \mathrm{~km} \mathrm{~s}^{-1}$ to $5000 \mathrm{~km} \mathrm{~s}^{-1}$ were listed until the cutoff date. Morphological information was given in the catalogues for $3276(50 \%)$ galaxies. We visually inspected all these galaxies using ALADIN2.5 software.

The arm patterns (S- or Z-type) of the galaxies were studied visually by the author in order to maintain homogeneity. The contour maps of the galaxies were studied in order to 
Table 1 The list of catalogues used for the data compilation. The first column lists the abbreviation of the catalogue. The second column gives the total number of galaxies. The references are listed in the last column.

\begin{tabular}{lrl}
\hline Catalogue & N References \\
\hline NGC & 623 Dreyer (1895, 1908) \\
UGC & 276 Nilson (1973) \\
ESO & 123 Lauberts (1982) \\
IC & 93 Dreyer (1895, 1908) \\
MCG & 88 Vorontsov-Vel'Yaminov et al. \\
& (1962-74) \\
UGCA & 84 Nilson (1974) \\
CGCG & 75 Zwicky et al. (1961-68) \\
KUG & 44 Takase (1980-2000) \\
MRK & 40 Markarian (1967) \\
MESSIER & 41 Messier (1784) \\
BCG & 28 Brandner et al. (2000) \\
LSBG & 23 Impey et al. (1996) \\
SBS & 17 Markarian et al. (1983) \\
LCRS & 16 Shectman et al. (1996) \\
DDO & 15 Bergh (1959, 1966) \\
IRAS & 15 Infrared Astronomical Satellite \\
& & (1983) \\
SGC & 10 Corwin et al. (1985) \\
UM & 10 MacAlpine et al. \\
& & (1977a,b,c; 1978; 1981) \\
\hline
\end{tabular}

identify their structural modes. For this, we used ALADIN2.5 software. The Z-mode is one whose outer tip points towards the anti-clockwise direction (see Fig. 1a). Similarly, the outer tip of S-mode directs in the clockwise direction. These two patterns are obviously the two dimensional projections of three dimensional galaxy. The re-examination of the S- and Z-modes using MIDAS software resulted the rejection of more than $17 \%$ of the objects. These rejected galaxies were nearly edge-on spiral and barred spiral galaxies. As expected, it was relatively easier to identify the structural modes of nearly face-on than that of nearly edge-on galaxies.

In this way, we compiled a database of 1621 galaxies showing either S- or Z-structural mode. There were 807 Z-mode and 821 S-mode galaxies in our database. All sky distribution of Zand S-mode galaxies is shown in Fig. 1b. The symbols "०" and " $\triangle$ " represent the positions of the S-mode and Z-mode galaxies, respectively. Several groups and aggregations of the galaxies can be seen in the figure.

The morphology, radial velocity, axial ratio and the magnitude distributions of S- and Zmode galaxies are shown in Figs. 1c,d,e,f. The spirals (47\%) dominate our database (Fig. 1c). However, a significant dominance of S-modes are noticed in the barred spirals whereas a weak dominance of Z-modes are found in the spirals. The population of galaxies in the RV distribution $\left(\Delta \mathrm{RV}=500 \mathrm{~km} \mathrm{~s}^{-1}\right)$ were nearly equal (Fig. 1d). The axial ratio distribution shows a good agreement with the expected cosine curve in the limit $0.2<b / a \leq 0.9$ (Fig. 1e). The values of absolute magnitude lie between 13.0 and 16.0 for $82 \%$ galaxies in our database (Fig. 1f).

We classified the database into 32 subsamples for both the S- and Z-modes on the basis of the morphology, radial velocity, area and the group of the galaxies. The galaxies with doubtful morphology are omitted in the spiral and barred spiral subsamples. The total number of earlyand late-type spirals or barred spirals is much less than that of the total spiral or total barred spirals. It is because of the fact that the galaxy with incomplete morphology, say, simply ' $\mathrm{S}$ ' or 'SB' can not be included in the subsamples. In other words, the galaxy with morphology Sa, $\mathrm{Sab}, \mathrm{Sb}, \mathrm{Sbc}$ are included in the early spirals whereas the galaxies with morphology Sc, Scd, Sd and Sdm are classified as late type spirals. The galaxies having morphology other than Sa, Sab, 
$\mathrm{Sb}, \mathrm{Sbc}, \mathrm{Sc}, \mathrm{Scd}, \mathrm{Sd}$ and Sm can not be included in the early and late subsamples. A statistical study of these subsamples are given in Table 1 and discussed in Sect. 4.1.

\section{METHOD OF ANALYSIS}

Basic statistics is used to study the dominance of Z- or S-mode galaxies. At first, morphology and RV dependence are studied. Secondly, sky is divided into 16 equal parts in order to observe deviation from the homogeneity. Several galaxy groups are identified in the all-sky map where the structural dominance are noticed. Finally, we study the dominance of Z- or S-mode galaxies in these groups.

We assume isotropic distribution as a theoretical reference and studied the equatorial PAdistribution in the total sample and subsamples. In order to measure the deviation from isotropic distribution we have carried out three statistical tests: chi-square, auto-correlation and the Fourier.

We set the chi-square probability $\mathrm{P}\left(>\chi^{2}\right)=0.050$ as the critical value to discriminate isotropy from anisotropy, this corresponds to a deviation from isotropy at the $2 \sigma$ level (Godlowski 1993). Auto correlation test takes account the correlation between the number of galaxies in adjoining angular bins. We expect, auto correlation coefficient $\mathrm{C} \rightarrow 0$ for an isotropic distribution. The critical limit is the standard deviation of the correlation coefficient C.

If the deviation from isotropy is only slowly varying with angles (in our case: PA) the Fourier test can be applied (Godlowski 1993). A method of expanding a function by expressing it as an infinite series of periodic functions (sine and cosine) is called Fourier series. Let $N$ denote the total number of solutions for galaxies in the sample, $N_{k}$ the number of solutions in the $\mathrm{k}^{\text {th }}$ bin, $N_{0}$ the mean number of solutions per bin, and $N_{0 k}$ the expected number of solutions in the $\mathrm{k}^{\text {th }}$ bin. Then the Fourier series is given by (taking first order Fourier mode),

$$
N_{k}=N_{k}\left(1+\Delta_{11} \cos 2 \beta_{k}+\Delta_{21} \sin 2 \beta_{k}+\ldots \ldots\right)
$$

Here the angle $\beta_{k}$ represents the polar angle in the $\mathrm{k}^{\text {th }}$ bin. The Fourier coefficients $\Delta_{11}$ and $\Delta_{21}$ are the parameters of the distributions. We obtain the following expressions for the Fourier coefficients $\Delta_{11}$ and $\Delta_{21}$,

$$
\begin{aligned}
& \Delta_{11}=\sum\left(N_{k}-N_{0 k}\right) \cos 2 \beta_{k} / \sum N_{0 k} \cos ^{2} 2 \beta_{k} \\
& \Delta_{21}=\sum\left(N_{k}-N_{0 k}\right) \sin 2 \beta_{k} / \sum N_{0 k} \sin ^{2} 2 \beta_{k}
\end{aligned}
$$

The standard deviations $\left(\sigma\left(\Delta_{11}\right)\right)$ and $\left(\sigma\left(\Delta_{21}\right)\right)$ can be estimated using the expressions,

$$
\begin{aligned}
& \sigma\left(\Delta_{11}\right)=\left(\sum N_{0 k} \cos ^{2} 2 \beta_{k}\right)^{-1 / 2} \\
& \sigma\left(\Delta_{21}\right)=\left(\sum N_{0 k} \sin ^{2} 2 \beta_{k}\right)^{-1 / 2}
\end{aligned}
$$

The probability that the amplitude

$$
\Delta_{1}=\left(\Delta_{11}^{2}+\Delta_{21}^{2}\right)^{1 / 2}
$$

greater than a certain chosen value is given by the formula

$$
P\left(>\Delta_{1}\right)=\exp \left(-n N_{0} \Delta_{1}^{2} / 4\right)
$$

with standard deviation

$$
\sigma\left(\Delta_{1}\right)=\left(2 / n N_{0}\right)^{1 / 2}
$$

The Fourier coefficient $\Delta_{11}$ gives the direction of departure from isotropy. The first order Fourier probability function $P\left(>\Delta_{1}\right)$ estimates whether (smaller value of $P\left(>\Delta_{1}\right)$ or not (higher value of $P\left(>\Delta_{1}\right)$ a pronounced preferred orientation occurs in the sample. 


\section{RESULTS}

First we present the statistical result concerning the distribution of Z- and S-mode galaxies in the total sample and subsamples. Second, we study the distribution of Z- and S-mode galaxies in the unit area of the sky and in the groups. Then, the equatorial PA-distribution of galaxies in the total sample and subsamples are discussed. At the end, a general discussion and a comparison with the previous results will be presented.

\subsection{Distribution of $\mathrm{Z}$ and $\mathrm{S}$ mode galaxies}

A statistical comparison between the total sample and subsamples of the Z- and S-modes of galaxies is given in Table 2. Fig. 2 shows this comparison in the histogram. The $\Delta(\%)$ in Table 1 and Fig. 2 represent the percentage difference between the number Z- and S-mode galaxies. We studied the standard deviation of the major diameters $(a)$ of galaxies in the total sample and subsamples for both the Z- and S-modes. In Table $2, \Delta(a$ sde $)$ represents the difference between the standard deviation of the major diameters of Z- and S-mode galaxies.

An insignificant difference $(0.4 \% \pm 0.2 \%)$ between the total number of Z- and S-mode galaxies are found (Table 2). The difference between the standard deviation of the major diameters $(\Delta$ (a sde $))$ of the Z- and S-mode galaxies is found less than 0.019 (eighth column, Table 2). Interestingly, the sum of the major diameters of total Z- and S-mode galaxies coincide. This result suggests the homogeneous distribution of Z- and S-mode field galaxies that have RV in the range $3000 \mathrm{~km} \mathrm{~s}^{-1}$ to $4000 \mathrm{~km} \mathrm{~s}^{-1}$.

In Fig. 2, the slanting-line (grey-shaded) region corresponds to the region showing $\leq 10 \%$ (5\%) $\Delta$ value. Almost all subsamples lie in this region, suggesting the homogeneous distribution of Z- and S-mode galaxies within 10\% error limit. Now, we present the distribution Z- and Smode galaxies in the subsamples classified according as their morphology, RVs, area and the groups below.

\subsubsection{Morphology}

In the spirals, Z-mode galaxies are found 3.7\% $( \pm 1.8 \%)$ more than that of S-mode. The homogeneous distribution of Z- and S-modes is found for the late-type spirals (Sc, Scd, Sd and Sm) than that of early-type (Sa, Sab, Sb and Sbc): $\Delta$ value turned out to be $9.5 \%( \pm 4.8 \%)$ and $1.8 \%( \pm 1.0 \%)$ for early- and late-types (Table 2$)$. Thus, no preferred winding pattern is noticed in the late-type spirals than that of early-types.

A significant dominance of S-mode galaxies are noticed $(17 \% \pm 8.5 \%)$ in spiral barred galaxies. The $\Delta$ value is found $>9 \%$ for both early- (SBa, SBab, SBb and SBbc) and late-type (SBc, $\mathrm{SBcd}, \mathrm{SBd}, \mathrm{SBm}$ ) barred spirals. Similar result (i.e., $\Delta>8 \%$ ) is found for the irregulars and the morphologically unidentified galaxies.

A similarity is noticed between the late-type spirals and barred spirals: the $\Delta$ value for both the late-types are found to be less than that of early-types (see Table 2).

The difference between the standard deviation of the major diameters $(\Delta(a$ sde $))$ for Sand Z-mode galaxies is found less than 0.050 arc minute for the total sample, spirals and the late-type spirals (eighth column, Table 1). These samples showed $\Delta$ value $<5 \%$ (grey-shaded region, Fig. 2a). Thus, we noticed a good correlation between the $\Delta(\%)$ and $\Delta(a$ sde $)$ value.

The difference between the sum of the major diameters (in percentage) are found greater than $10 \%$ for the barred spirals and early-type barred spirals. Interestingly, these two subsamples showed $\Delta$ value greater than $15 \%$ (Fig. 2a). Thus, inhomogeniety in the distribution of S- and Z-mode galaxies is noticed for barred spirals. 

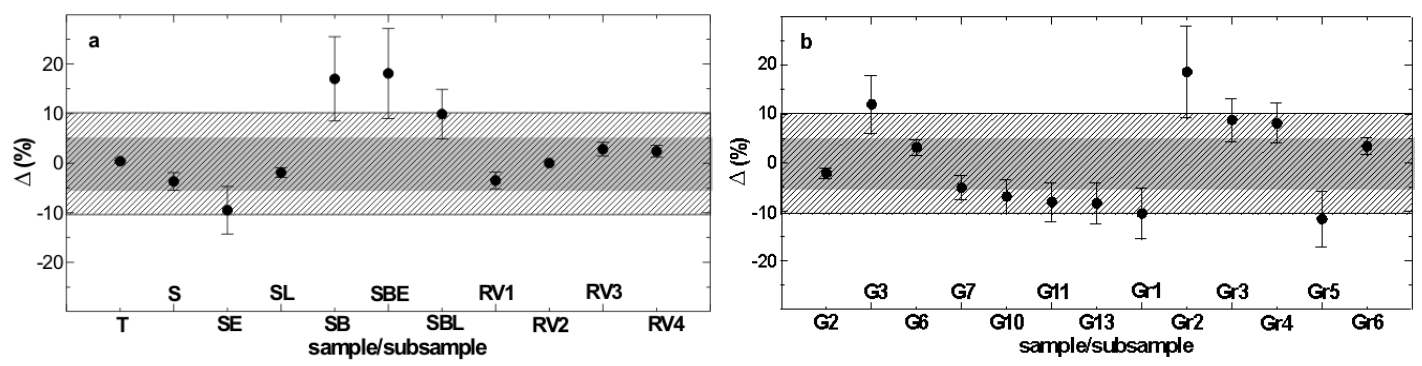

Fig. 2 The basic statistics of the $\mathrm{Z}$ and S-mode of galaxies in the total sample and subsamples. The full form of the abbreviations (X-axis) are given in Table 2 (first column). $\Delta(\%)=S-Z$, where $S$ and $Z$ represent the number of S- and Z-mode galaxies, respectively. The statistical error bars $\sigma(\%)$ shown in the figure are calculated as: $\sigma(\%)$ $=\sigma /(\sqrt{S}+\sqrt{Z}) \times 100$, where $\sigma=(\sqrt{S}-\sqrt{Z})$. The grey-shaded and the slanting-line region represent the $\leq \pm 5 \%$ and $\leq \pm 10 \% \Delta$ value, respectively.

\subsubsection{Radial velocity}

A very good correlation between the number of S- and Z-mode can be seen in the RV classifications (Fig. 1d). All 4 subsamples show the $\Delta$ and $\Delta(a$ sde) values less than $5 \%$ and 0.050 , respectively (Table 2). In addition, $\Delta(a)$ is found to be $<5 \%$. This result is important in the sense that the statistics in these subsamples is rich (number of galaxies $>170$ ) enough. Thus, we could not observe preference structural modes ( $\mathrm{S}$ or $\mathrm{Z}$ ) in the low and high RV galaxies in our database.

A difference is noticed: dominance of Z- and S- modes, respectively in low (RV1) and high (RV3, RV4) RV subsamples. However, this dominance is not significant (i.e., $\Delta<5 \%$ ). An equal number of S- and Z-mode galaxies are found in the subsample RV2 $\left(3500<\mathrm{RV}\left(\mathrm{km} \mathrm{s}^{-1}\right) \leq\right.$ 4000 ) (Table 1). In order to check the binning effect, we further classify the total galaxies in 6 $\left(\Delta \mathrm{RV}=333 \mathrm{~km} \mathrm{~s}^{-1}\right)$ and 8 bins $\left(\Delta \mathrm{RV}=250 \mathrm{~km} \mathrm{~s}^{-1}\right)$ and study the statistics. No significant dominance of either S- and Z-modes are noticed.

Thus, it is found that the homogeneous distribution of S- and Z-mode galaxies remain invariant with the global expansion (i.e, expansion of the Universe). We further discuss this result below.

\subsubsection{Area}

We study the distribution of S- and Z-mode galaxies by dividing the sky into 16 equal parts (Fig. 3a). The area of the grid $(\mathrm{G})$ is $90^{\circ} \times 45^{\circ}(\mathrm{RA} \times \mathrm{Dec})$. The area distribution of S- and Z-mode galaxies are plotted, that can be seen in Fig. 3a'. The statistical parameters are given in Table 2.

A significant dominance $(>2 \sigma)$ of S-mode is noticed in grid 3 (RA: $0^{\circ}$ to $90^{\circ}$, Dec: $0^{\circ}$ to $45^{\circ}$ (J2000)) (Fig. 3a, a'). An elongated group of galaxies can be seen in this grid. In this grid, $\Delta, \Delta(a$ sde) and $\Delta(a) \%$ are found to be $12 \% \pm 6 \%, 0.076$ and $9.3 \%$, respectively. These figures suggest that the distribution of S- and Z-mode galaxies in G3 is not homogeneous. Probably, this is due to the apparent subgroupings or subclusterings of the galaxies.

The S-mode galaxies dominate in the grids G8 and G9 (Fig. 3a'). However, the statistics is poor $(<40)$ in these grids (Table 2$)$. In addition, no groupings or subclustering are noticed.

A dominance $(\sim 1.5 \sigma)$ of Z-mode is noticed in G10 (RA: $180^{\circ}$ to $270^{\circ}$, Dec: $-45^{\circ}$ to $0^{\circ}$ (J2000)) and G13 (RA: $270^{\circ}$ to $360^{\circ}$, Dec: $-90^{\circ}$ to $-45^{\circ}$ (J2000)) (Fig. 3a,a'). In both the grids, a large aggregation of the galaxies can be seen. A subcluster-like aggregation can be seen in 
Table 2 Statistics of leading (column 3) and trailing arm (column 4) galaxies in the total sample and subsamples. The fifth and sixth column give the numeral and percentage difference $(\Delta=S-Z)$ between the S- $(S)$ and the Z- $(Z)$ modes. The next two columns give the error: $\sigma=(\sqrt{S}-\sqrt{Z})$ and $\sigma(\%)=\sigma /(\sqrt{S}+\sqrt{Z}) \times 100$. The eighth column gives the difference between the standard deviation (in arcmin) of the major diameters $(a)$ of the S- and Z-modes galaxies $(\Delta($ a sde $))$. The difference between the sum of the major diameters $(\Delta(a) \%)$ are listed in the last column. The sample/subsample and their abbreviations are given in first two columns.

\begin{tabular}{|c|c|c|c|c|c|c|c|c|}
\hline sample/subsample & symbol & $\mathrm{Z}$ & $\mathrm{S}$ & $\Delta$ & $\Delta(\%)$ & $\sigma(\%)$ & $\Delta(a$ sde $)$ & $\Delta(a)(\%)$ \\
\hline Total & $\mathrm{T}$ & 814 & 807 & -7 & -0.4 & -0.2 & 0.019 & 0.0 \\
\hline Spiral & S & 395 & 367 & -28 & -3.7 & -1.8 & 0.031 & 3.0 \\
\hline Spiral (early-type) & SE & 150 & 124 & -26 & -9.5 & -4.8 & 0.058 & 8.1 \\
\hline Spiral (late-type) & SL & 131 & 126 & -5 & -1.9 & -1.0 & 0.031 & 0.3 \\
\hline Barred Spiral & SB & 191 & 269 & 78 & 17.0 & 8.5 & 0.062 & 15.2 \\
\hline Barred Spiral (early-type) & SBE & 97 & 140 & 43 & 18.1 & 9.1 & 0.091 & 14.6 \\
\hline Barred Spiral (late-type) & SBL & 68 & 83 & 15 & 9.9 & 5.0 & 0.066 & 9.2 \\
\hline $3000<\mathrm{RV}\left(\mathrm{km} \mathrm{s}^{-1}\right) \leq 3500$ & RV1 & 208 & 194 & -14 & -3.5 & -1.7 & 0.046 & 2.6 \\
\hline $3500<\mathrm{RV}\left(\mathrm{km} \mathrm{s}^{-1}\right) \leq 4000$ & RV2 & 201 & 201 & 0 & 0.0 & 0.0 & 0.031 & 3.1 \\
\hline $4000<\mathrm{RV}\left(\mathrm{km} \mathrm{s}^{-1}\right) \leq 4500$ & RV3 & 172 & 182 & 10 & 2.8 & 1.4 & 0.034 & 0.6 \\
\hline $4500<\mathrm{RV}\left(\mathrm{km} \mathrm{s}^{-1}\right) \leq 5000$ & RV4 & 226 & 237 & 11 & 2.4 & 1.2 & 0.041 & 1.0 \\
\hline Grid 1 & G1 & 20 & 21 & 1 & 2.4 & 1.2 & 0.068 & 6.8 \\
\hline Grid 2 & G2 & 121 & 116 & -5 & -2.1 & -1.1 & 0.014 & 1.6 \\
\hline Grid 3 & G3 & 88 & 112 & 24 & 12.0 & 6.0 & 0.076 & 9.3 \\
\hline Grid 4 & G4 & 12 & 14 & 2 & 7.7 & 3.9 & 0.647 & 9.7 \\
\hline Grid 5 & G5 & 11 & 8 & $-\overline{3}$ & -15.8 & -7.9 & 0.042 & 22.3 \\
\hline Grid 6 & G6 & 75 & 80 & 5 & 3.2 & 1.6 & 0.081 & 4.5 \\
\hline Grid 7 & G7 & 62 & 56 & -6 & -5.1 & -2.5 & 0.095 & 8.9 \\
\hline Grid 8 & G8 & 22 & 33 & 11 & 20.0 & 10.1 & 0.073 & 12.9 \\
\hline Grid 9 & G9 & 20 & 31 & 11 & 21.6 & 10.9 & 0.028 & 18.1 \\
\hline Grid 10 & G10 & 124 & 108 & -16 & -6.9 & -3.5 & 0.004 & 5.4 \\
\hline Grid 11 & G11 & 61 & 52 & -9 & -8.0 & -4.0 & 0.025 & 6.2 \\
\hline Grid 12 & G12 & 20 & 20 & 0 & 0.0 & 0.0 & 0.409 & 7.4 \\
\hline Grid 13 & G13 & 78 & 66 & -12 & -8.3 & -4.2 & 0.039 & 2.9 \\
\hline Grid 14 & G14 & 37 & 44 & 7 & 8.6 & 4.3 & 0.356 & 10.3 \\
\hline Grid 15 & G15 & 47 & 44 & -3 & -3.3 & -1.6 & 0.050 & 5.2 \\
\hline Grid 16 & G16 & 9 & 9 & 0 & 0.0 & 0.0 & 0.191 & 8.6 \\
\hline Group 1 & Gr1 & 37 & 30 & -7 & -10.4 & -5.2 & 0.032 & 7.1 \\
\hline Group 2 & Gr2 & 48 & 70 & 22 & 18.6 & 9.4 & 0.097 & 12.6 \\
\hline Group 3 & Gr3 & 31 & 37 & 6 & 8.8 & 4.4 & 0.027 & 4.3 \\
\hline Group 4 & Gr4 & 34 & 40 & 6 & 8.1 & 4.1 & 0.031 & 3.9 \\
\hline Group 5 & Gr5 & 107 & 85 & -22 & -11.5 & -5.7 & 0.089 & 11.2 \\
\hline Group 6 & Gr6 & 42 & 45 & 3 & 3.4 & 1.7 & 0.024 & 1.6 \\
\hline
\end{tabular}

G10. An elongated structure can be seen in G13. In both the grids, $\Delta$ value is found to be greater than $5 \%$ (Table 2).

No dominance of either S- and Z-mode galaxies is noticed in the groups G1, G2, G4, G5, G6, G7, G11, G12, G14, G15 and G16. Thus, homogeneous distribution of S- and Z-mode galaxies is found intact in $\sim 80 \%$ area of the sky. We suspect that the groupings or subclusterings of the galaxies lead the preference structural modes (S or Z) in G3, G10 and G13.

\subsubsection{Galaxy groups}

In all-sky map, several groups of galaxies can be seen (Fig. 3a). It is interesting to study the distribution of structural modes ( $\mathrm{S}$ or $\mathrm{Z}$ ) of galaxies in these groups. For this, we systematically searched for the groups fulfilling following selection criteria: (a) major diameter $>30^{\circ}$, (b) 

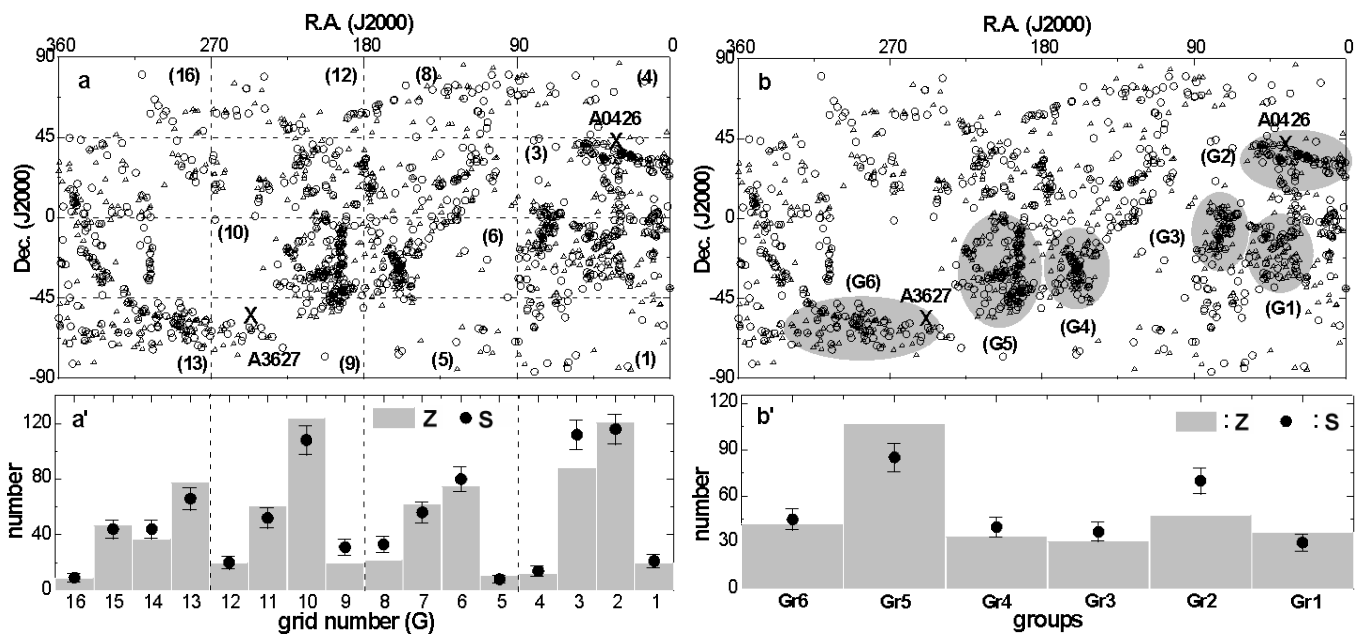

Fig. 3 (a) All sky distribution of Z-mode (hollow circle) and S-mode (hollow triangle) galaxies in 16 area grids. (a') The histogram showing the distribution of the Z- and S-mode galaxies in the grids G1 to G16. (b) Six groups of the galaxies, represented by the grey-shaded region. (b') The distribution of Z- and S-modes in 6 groups. The statistical error bar $\pm 1 \sigma$ is shown. The positions of the clusters Abell 0426 and Abell 3627 are shown by the symbol " $\times$ " $(\mathrm{a}, \mathrm{b})$.

cutoff diameter $<2$ times the background galaxies, (c) number of galaxies $>50$. We found 6 groups fulfilling these criteria (Fig. 3b). All 6 groups (Gr) are inspected carefully. In 3 groups (Gr2, Gr5 and Gr6), subgroups can be seen. The number of galaxies in the groups Gr2 and Gr5 are found more than 100 .

The clusters Abell 0426 and Abell 3627 are located close to the groups Gr2 and Gr6. The symbol " $\times$ " represents the position of the cluster center in Fig. 3b. The mean radial velocities of these clusters are $5366 \mathrm{~km} \mathrm{~s}^{-1}$ and $4881 \mathrm{~km} \mathrm{~s}^{-1}$, respectively. However, we have removed the member galaxies of these clusters from our database.

A significant dominance $(>2 \sigma)$ of S-mode galaxies is noticed in the group Gr2 (Fig. 3b,b'). The $\Delta, \Delta(a$ sde $)$ and $\Delta(a) \%$ values are found to be $18.6 \%( \pm 9.4 \%), 0.097$ and $12.6 \%$, respectively (Table 2). We suspect that the galaxies in this group is under the influence of the cluster Abell 0426, due to which apparent subclustering of the galaxies is seen.

The galaxies in Gr5 shows an opposite preference: a significant dominance of the Z-mode galaxies $(>2 \sigma)$ (Fig. 3b,b'). In this group, $\Delta, \Delta($ a sde) and $\Delta(a) \%$ are found to be $11.5 \% \pm$ $5.7 \%, 0.089$ and $11.2 \%$, suggesting inhomogeneous distribution of structural modes (Table 2).

No humps or dips can be seen in the groups Gr1, Gr3, Gr4 and Gr6 (Fig. 3.2b,b'). Thus, the distribution of S- and Z-mode galaxies in these groups are found to be homogeneous. The number of galaxies in these groups are less than 100 .

In the group 6, we could not notice the influence of the cluster Abell 3627. This might be due to the off location of the cluster center from the group center.

\subsection{Anisotropy in the position angle distribution}

We study the equatorial position angle (PA) distribution of S- and Z-mode galaxies in the total sample and subsamples. A spatially isotropic distribution is assumed in order to examine non-random effects in the PA-distribution. In order to discriminate the deviation from the randomness, we use three statistical tests: chi-square, auto correlation and the Fourier. The 


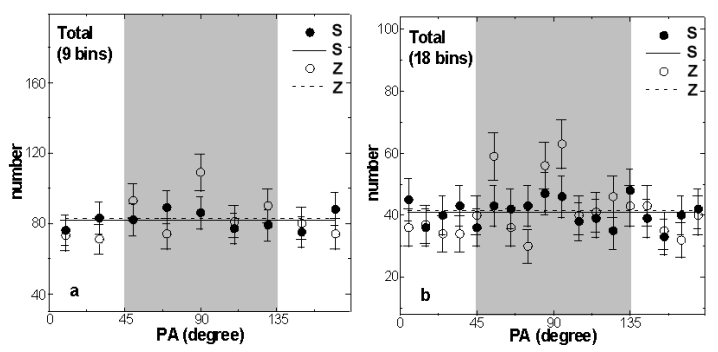

Fig. 4 The equatorial position angle (PA) distribution of total Z- and S-mode galaxies plotted in 9 (a) and 18 (b) bins. The solid and the dashed line represent the expected isotropic distribution for S- and Z-mode galaxies, respectively. The observed counts with statistical $\pm 1 \sigma$ error bars are shown. $\mathrm{PA}=90^{\circ} \pm 45^{\circ}$ (grey-shaded region) corresponds to the galactic rotation axes tend to be oriented perpendicular with respect to the equatorial plane.

bin size was chosen to be $20^{\circ}$ (9 bins) in all these tests. The statistically poor bins (number of solution $<5)$ are omitted in the analysis. The conditions for anisotropy are the following: the chi-square probability $\mathrm{P}\left(>\chi^{2}\right)<0.050$, correlation coefficient $C / \sigma(C)>1$, first order Fourier coefficient $\Delta_{11} / \sigma\left(\Delta_{11}\right)>1$ and the first order Fourier probability $\mathrm{P}\left(>\Delta_{1}\right)<0.150$ as used by Godlowski (1993). Table 3 lists the statistical parameters for the total samples and subsamples.

In the Fourier test, $\Delta_{11}<0$ (i.e., negative) indicates an excess of galaxies with the galactic planes parallel to the equatorial plane. In other words, a negative $\Delta_{11}$ suggests that the rotation axis of galaxies tend to be oriented perpendicular with respect to the equatorial plane. Similarly, $\Delta_{11}>0$ (i.e., positive) indicates that the rotation axis of galaxies tend to lie in the equatorial plane.

In the histograms (see Figs. 4-7), a hump at $90^{\circ} \pm 45^{\circ}$ (grey-shaded region) suggests that the galactic planes of galaxies tend to lie in the equatorial plane. In other words, the rotation axes of galaxies tend to be oriented perpendicular with respect to the equatorial plane when there is excess number of solutions in the grey-shaded region in the histogram.

All three statistical tests show isotropy in the total S-mode galaxies. Thus, no preferred alignment is noticed for the total S-mode galaxies (solid circles in Fig. 4a). Interestingly, all three statistical tests show anisotropy in the total Z-mode galaxies. The chi-square and Fourier probabilities $\left(\mathrm{P}\left(>\chi^{2}\right), \mathrm{P}\left(>\Delta_{1}\right)\right)$ are found $1.5 \%(<5 \%$ limit $)$ and $8.5 \%(<15 \%$ limit $)$, respectively (Table 2). The auto correlation coefficient $(\mathrm{C} / \mathrm{C}(\sigma))$ turned $-3.2(>>1)$. The $\Delta_{11} / \sigma\left(\Delta_{11}\right)$ value is found to be negative at $\sim 2 \sigma$ level, suggesting that the rotation axes of Z-mode galaxies tend to be oriented perpendicular the equatorial plane. Three humps at $50^{\circ}(>1.5 \sigma), 90^{\circ}(>2 \sigma)$ and $130^{\circ}(1.5 \sigma)$ support this result (Fig. 4a). We checked the biasness in the results due to bin size by increasing and decreasing the number of bins. A similar statistical result is found for both structural modes. Fig. 4b shows the PA-distribution histogram for the total sample in 18 bins. The Z-mode galaxies show three significant humps in the grey-shaded region, supporting the results mentioned above.

Thus, we conclude isotropy for S-mode whereas anisotropy for Z-mode galaxies in the total sample.

\subsubsection{Morphology}

In the spirals, the chi-square and auto correlation tests show isotropy for both the S- and Zmodes. The first order Fourier probability is found greater than 35\%, suggesting no preferred alignment. However, the $\Delta_{11}$ value exceeds $1 \sigma$ limit $(-1.2 \sigma)$ in the S-mode spirals. A hump at 
Table 3 Statistics of the PA-distribution of galaxies in the total sample and subsamples (first column). The second, third, fourth and fifth columns give the chi-square probability $\left(\mathrm{P}\left(>\chi^{2}\right)\right)$, correlation coefficient $(\mathrm{C} / \mathrm{C}(\sigma))$, first order Fourier coefficient $\left(\Delta_{11} / \sigma\left(\Delta_{11}\right)\right)$, and first order Fourier probability $\mathrm{P}\left(>\Delta_{1}\right)$, respectively. The last four columns repeats the previous columns.

\begin{tabular}{lcccccccc}
\hline sample & \multicolumn{3}{c}{ S-mode } & \multicolumn{7}{c}{ Z-mode } \\
& $\mathrm{P}\left(>\chi^{2}\right)$ & $\mathrm{C} / \mathrm{C}(\sigma)$ & $\Delta_{11} / \sigma\left(\Delta_{11}\right)$ & $\mathrm{P}\left(>\Delta_{1}\right)$ & $\mathrm{P}\left(>\chi^{2}\right)$ & $\mathrm{C} / \mathrm{C}(\sigma)$ & $\Delta_{11} / \sigma\left(\Delta_{11}\right)$ & $\mathrm{P}\left(>\Delta_{1}\right)$ \\
\hline total & 0.666 & +0.0 & -0.9 & 0.381 & 0.015 & -3.2 & -1.9 & 0.085 \\
S & 0.511 & -0.7 & -1.2 & 0.434 & 0.225 & +0.4 & +0.8 & 0.383 \\
SE & 0.973 & +0.1 & -0.9 & 0.569 & 0.031 & +2.0 & +2.8 & 0.015 \\
SL & 0.234 & +0.5 & +0.8 & 0.209 & 0.460 & -0.1 & -0.5 & 0.345 \\
SB & 0.729 & +0.3 & +1.0 & 0.454 & 0.285 & -1.0 & -0.2 & 0.497 \\
SBE & 0.739 & +0.1 & -0.5 & 0.566 & 0.230 & -0.7 & +0.1 & 0.521 \\
SBL & 0.043 & +1.8 & +1.7 & 0.046 & 0.620 & -0.9 & -0.2 & 0.872 \\
RV1 & 0.910 & +0.3 & +0.8 & 0.362 & 0.369 & -0.9 & -0.6 & 0.285 \\
RV2 & 0.790 & +0.3 & -1.0 & 0.496 & 0.925 & -0.4 & -0.2 & 0.887 \\
RV3 & 0.050 & +1.6 & -1.5 & 0.083 & 0.033 & -1.8 & -2.3 & 0.046 \\
RV4 & 0.043 & -2.3 & -1.5 & 0.116 & 0.636 & +0.2 & -0.7 & 0.692 \\
Gr2 & 0.455 & +0.6 & +0.8 & 0.861 & 0.033 & -1.8 & +1.7 & 0.116 \\
Gr5 & 0.033 & -1.4 & -2.0 & 0.085 & 0.516 & +0.4 & -0.4 & 0.548 \\
\hline
\end{tabular}
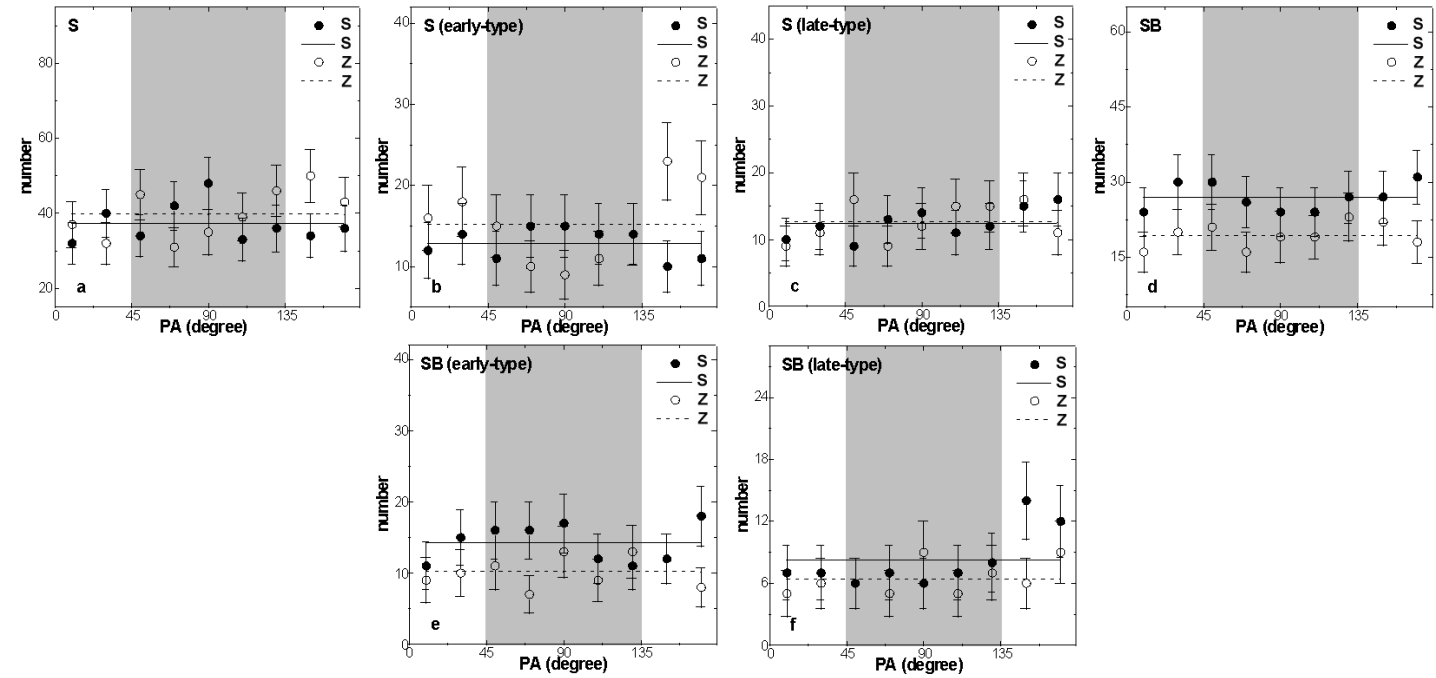

Fig. 5 The equatorial PA-distribution of Z- and S-mode galaxies in the spirals (a), early-type spirals (b), late-type spirals (c), barred spirals (d), early-type barred spirals (e) and late-type barred spirals (f). The symbols, error bars, dashed lines and the explanations are analogous to Fig. 4.

$90^{\circ}$ is not enough to turn the $\Delta_{11} / \sigma\left(\Delta_{11}\right)>1.5$ (Fig. 5a). Similarly, a hump at $150^{\circ}$ is not enough to make the $\Delta_{11} / \sigma\left(\Delta_{11}\right)>1.5$ in the Z-mode spirals. Hence, the preferred alignment is not profounded in both the S- and Z-mode spirals. Thus, we conclude a random orientation of S- and Z-mode spirals. 

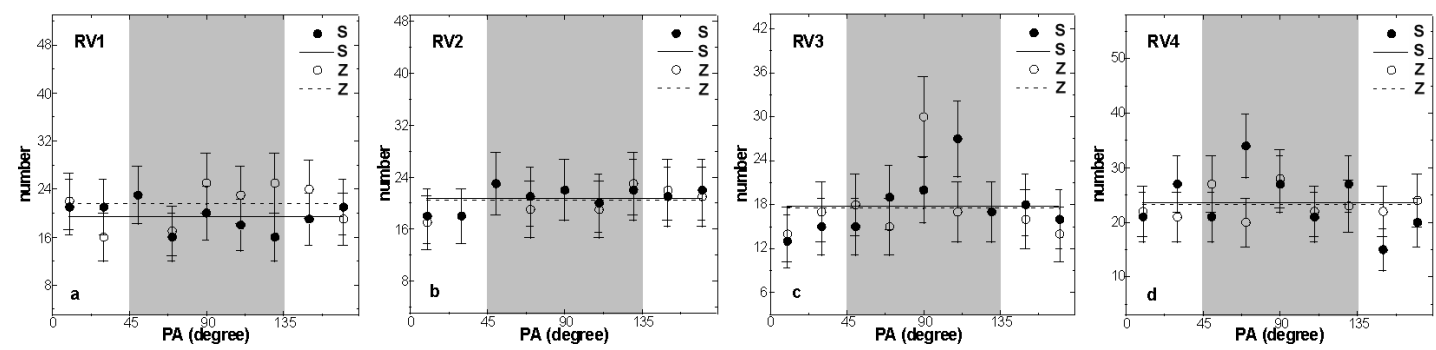

Fig. 6 The equatorial PA-distribution of Z- and S-mode galaxies in RV1 (a), RV2 (b), RV3 (c) and RV4 (d). The abbreviations are listed in Table 1. The symbols, error bars, dashed lines and the explanations are analogous to Fig. 4.

Early- and late-type S-mode spirals show isotropy in all three statistical tests (Table 3). No humps and the dips are seen in the histograms (solid circles in Fig. 5b,c). Thus, the Smode spirals show a random alignment in the PA-distribution. In the subsample SE, all three statistical tests show anisotropy (Table 2). Two significant humps at $>150^{\circ}$ cause the first order Fourier coefficient $\left(\Delta_{11}\right)>+2.5 \sigma$ (hollow circle in Fig. $5 \mathrm{~b}$ ). Thus, a preferred alignment is noticed in the early-type Z-mode spirals: the galactic rotation axes tend to lie in the equatorial plane. The late-type Z-mode spirals show a random alignment.

The spiral barred galaxies show a random alignment in both the S- and Z-modes. In Fig. $5 \mathrm{~d}$, no deviation from the expected distribution can be seen. All three statistical tests support this result (Table 2). A similar result is found for the early-type SB galaxies in both structural modes (Table 3, Fig. 5e).

The $\mathrm{P}\left(>\chi^{2}\right)$ and $\mathrm{P}\left(>\Delta_{1}\right)$ are found less than $5 \%$, suggesting a preferred alignment for the late-type S-mode SB galaxies (Table 3$)$. The auto correlation coefficient $(\mathrm{C} / \mathrm{C}(\sigma))$ and the hump at $>150^{\circ}$ support this result (Fig. 5f). The $\Delta_{11} / \sigma\left(\Delta_{11}\right)$ is found to be positive at $1.7 \sigma$ level, suggesting that the S-mode SBL galaxies tend to lie in the equatorial plane. Thus, the late-type S- and Z-mode SB galaxies show preferred and random alignments, respectively.

\subsubsection{Radial velocity}

The subsamples RV1 and RV2 show isotropy in all three statistical tests (Table 2). No humps or dips can be seen in Figs. 6a,b. Thus, the galaxies having radial velocity in the range 3000 $\mathrm{km} \mathrm{s}^{-1}$ to $4000 \mathrm{~km} \mathrm{~s}^{-1}$ show a random alignment for both the S- and Z-mode galaxies.

The humps at $90^{\circ}(>2 \sigma)$ and $110^{\circ}(>2 \sigma)$ are found in the Z- and S-mode RV3 galaxies, respectively (Fig. 6c). These two significant humps lead the subsample to show anisotropy in the statistical tests (Table 2). The $\Delta_{11}$ values are found negative at $\geq 1.5$ level, suggesting a similar preferred alignment for both modes: the galaxy rotation axes tend to be directed perpendicular to the equatorial plane.

A hump at $70^{\circ}(>1.5 \sigma)$ and a dip at $150^{\circ}(\sim 2 \sigma)$ cause the S-mode RV4 galaxies to show anisotropy in all three statistical tests (Fig. 6d). Thus, the S-mode galaxies having radial velocity in the range $4500 \mathrm{~km} \mathrm{~s}^{-1}$ to $5000 \mathrm{~km} \mathrm{~s}^{-1}$ show a similar preferred alignments as shown by the subsample RV3: galactic planes of galaxies tend to lie in the equatorial plane. The leading arm galaxies in the subsample RV4 show a random alignment (Table 3, Fig. 6d).

\subsubsection{Groups}

We do not study PA-distribution of S- and Z-mode galaxies in the groups Gr1, Gr3, Gr4 and Gr6 because of poor statistics (number $<50$ ). 

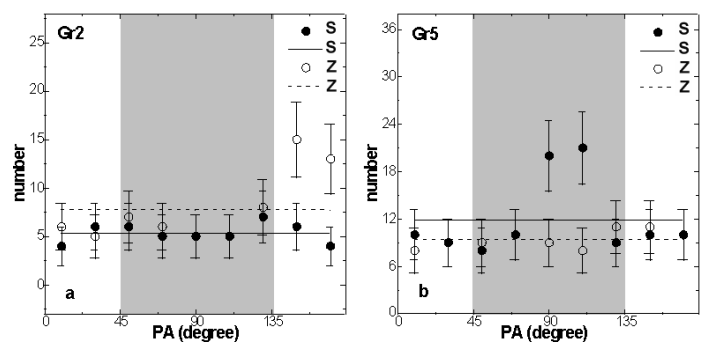

Fig. 7 The equatorial PA-distribution of Z- and S-mode galaxies in the groups Gr2 and Gr5. The abbreviations are listed in Table 1. The symbols, error bars, dashed lines and the explanations are analogous to Fig. 4.

We study the PA-distribution of S- and Z-mode galaxies in the groups Gr2 and Gr5, where the dominance of either Z- or S-mode is noticed. In addition, the statistics is relatively better in these groups.

In the group Gr2, Z-mode galaxies dominate the S-mode galaxies. In this group, the Z-mode galaxies show a preferred alignment whereas S-mode galaxies show a random alignment in the PA-distribution. All three statistical tests suggest anisotropy in the Z-mode galaxies (Table 3). The humps at $>150^{\circ}$ cause the $\Delta_{11}$ value to be positive at $>1.5 \sigma$ level (Fig. 7a), suggesting that the rotation axes of Z-mode galaxies in $\mathrm{Gr} 2$ tend to be oriented parallel the equatorial plane.

The S-mode galaxies dominate in the group Gr5. Interestingly, a preferred alignment of S-mode galaxies is noticed in the PA-distribution. In Fig. $7 \mathrm{~b}$, two significant humps at $90^{\circ}$ $(\sim 2 \sigma)$ and $110^{\circ}(>2 \sigma)$ can be seen. These humps lead the subsample (S-mode Gr5) to show anisotropy in the statistical tests (Table 3). No preferred alignment is noticed in the Z-mode galaxies of this group.

Thus, the dominating structural modes ( $\mathrm{Z}$ or $\mathrm{S}$ ) show a preferred alignment in the PAdistribution. This is noticeable result.

\subsection{Discussion}

Fig. 8 shows a comparison between the number $(\Delta)$ and position angle $\left(\Delta_{11} / \sigma\left(\Delta_{11}\right)\right)$ distribution of S- and Z-mode galaxies in the total sample and subsamples. This plot deals the correlation between the homogeneity in the structural modes and the random alignment in the subsamples. The grey-shaded region represents the region of isotropy and homogeneity for the $\Delta_{11} / \sigma\left(\Delta_{11}\right)$ and $\Delta(\%)$, respectively. Twenty five (out of $39,64 \%$ ) subsamples lie in the greyshaded region (Fig. 8a), suggesting a good agreement between the homogeneous distribution of S- and Z-mode galaxies and the random alignment of the rotation axes of galaxies. In four subsamples (SE, SBL, Gr2 and Gr5), a good correlation between the preferred alignment and the dominance of either S- or Z-mode galaxies is noticed (Fig. 8a). Thus, it is noticed that the random alignment of the PAs of galaxies hint the existence of inhomogeneity in the structural modes.

Aryal \& Saurer (2006) and Aryal, Paudel \& Saurer (2007) studied the spatial orientation of galaxies in 32 Abell clusters of BM type I, II, II-III and III and found a significant preferred alignment in the late-type cluster (BM type II-III, III). They concluded that the randomness decreases systematically in galaxy alignments from early-type (BM type I, II) to late-type (BM type II-III, III) clusters.

We noticed a very good correlation between the random alignments and the homogeneity in the structural modes. Probably, this result reveals the fact that the progressive loss of homo- 


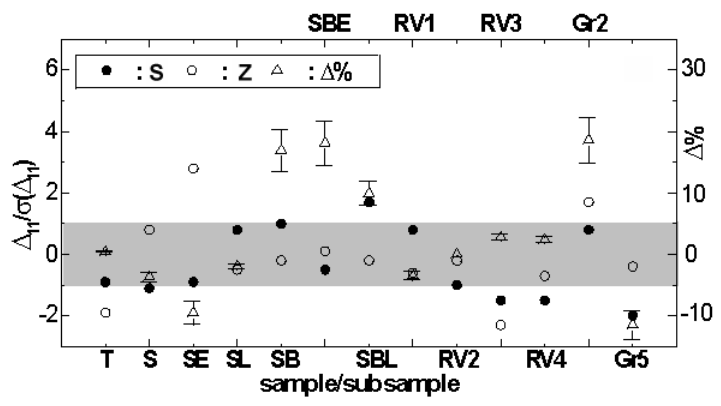

Fig. 8 A comparison between the number $(\Delta \%)$ and the position angle $\left(\Delta_{11} / \sigma\left(\Delta_{11}\right)\right)$ distribution of Z- and S-mode galaxies in the total sample and subsamples.

geneity in the structural modes might have some connection with the rotationally supported (spirals, barred spirals) to the randomized (lenticulars, ellipticals) system. Thus, we suspect that the dynamical processes in the cluster evolution (such as late-type clusters) give rise to a dynamical loss of homogeneity in the structural modes. It would be interesting to test this prediction by studying the S- and Z-type spirals in the late-type clusters in the future.

As $60 \%$ of galaxies in the nearby universe are rotationally supported discs, understanding angular-momentum acquisition is obviously a crucial part of understanding galaxy evolution. The winding sense of spiral arm patterns (morphological feature) allows us to infer the orientation of the angular-momentum vector of the disc galaxy. The expected distribution of spin vectors of galaxies shows markedly different trends according to the galaxy formation scenarios. One can suspect the possibility that the actual distribution of galaxy spin shows a dipole or a quadrupole component depending on the scenarios of galaxy formation. If galaxy spins were generated according to the primordial whirl scenario, a strong bias in either the $\mathrm{S}$ or $\mathrm{Z}$ patterns would be seen in a face-on sample of galaxies. We did not find this trend in our sample. A quadrupole distribution of $\mathrm{S} / \mathrm{Z}$ might be observed if the primary process was the generation of spins due to the pancake shock scenario or the explosion scenario. On the other hand, if the galaxy spins were produced by the tidal spin-up process, there would be no global anisotropy as we noticed in many cases, unless galaxy-cluster tidal interaction rather than galaxy-galaxy tidal interaction were the primary process. No significant correlation, however, was identified in any ensemble.

\section{CONCLUSIONS}

We studied the winding sense of 1621 field galaxies around the Local Supercluster. These galaxies have radial velocity (RV) in the range $3000 \mathrm{~km} \mathrm{~s}^{-1}$ to $5000 \mathrm{~km} \mathrm{~s}^{-1}$. The distribution of Z- and S-mode galaxies is studied in the total sample and 32 subsamples. To examine nonrandom effects, the equatorial position angle $(\mathrm{PA})$ distribution of galaxies in the total sample and subsamples are studied. In order to discriminate anisotropy from the isotropy we have performed three statistical tests: chi-square, auto-correlation and the Fourier. Our results are as follows:

1. The homogeneous distribution of the total Z- and S-mode galaxies is found, suggesting the homogeneous distribution of winding sense ( $\mathrm{S}$ or Z) of galaxies having RVs $3000 \mathrm{~km} \mathrm{~s}^{-1}$ to $5000 \mathrm{~km} \mathrm{~s}^{-1}$. The PA-distribution of S-mode galaxies is found to be random, whereas preferred alignment is noticed for Z-mode galaxies. It is found that the galactic rotation axes of Z-mode galaxies tend to be oriented perpendicular the equatorial plane. 
2. Z-mode are found $3.7 \%( \pm 1.8 \%)$ more than that of the S-mode in the spirals whereas a significant dominance $(17 \% \pm 8.5 \%)$ of S-mode is noticed in the barred spirals. This difference is found $>8 \%$ for the irregulars and the morphologically unidentified galaxies. A random alignment is noticed in the PA-distribution of Z- and S-mode spirals. Thus, it is noticed that the random alignment of the PAs of galaxies lead the existence of inhomogeneity in the structural modes of galaxies.

3. The inhomogeneity in the structural modes is found stronger for the late-type spirals (Sc, $\mathrm{Scd}, \mathrm{Sd}$ and $\mathrm{Sm}$ ) than that of early-type (Sa, Sab, Sb and Sbc). Similar result is found for the late-type barred spirals.

4. A very good correlation between the number of Z- and S-mode galaxies are found in the RV subsamples. All 4 subsamples show the $\Delta$ value less than $5 \%$. Thus, we conclude that the homogeneous distribution of structural modes of field galaxies remain invariant with the global expansion.

5. The galaxies having RVs $3000 \mathrm{~km} \mathrm{~s}^{-1}$ to $4000 \mathrm{~km} \mathrm{~s}^{-1}$ show a random alignment for both the Z- and S-modes. The rotation axes of Z- and S-mode galaxies having $4000<\mathrm{RV}(\mathrm{km}$ $\left.\mathrm{s}^{-1}\right) \leq 4500$ tend to be oriented perpendicular the equatorial plane.

6. The distribution of the winding sense of galaxies is found homogeneous in $\sim 80 \%$ area of the sky. This property is found to be violated in few groups of galaxies. Two such groups (Gr2 and Gr8) are identified. In these groups, the structural dominance and the preferred alignments of galaxies are found to oppose each other.

The true structural mode of a galaxy must involve a determination of which side of the galaxy is closer to the observer (Binney and Tremaine 1987). Three-dimensional determination of the leading and the trailing arm patterns in the galaxies is a very important problem. We intend to address this problem in the future.

Acknowledgements We are indebted to the referee for his/her constructive criticism and useful comments. I acknowledge Profs. R. Weinberger and W. Saurer of Innsbruck University, Austria for insightful discussions. I am thankful to Tribhuvan University, Nepal and Innsbruck University, Austria for providing financial assistance to visit Innsbruck University during JanMarch 2009.

\section{References}

Abell, G.O., Corwin, H.G., Olowin, R.P. 1989, ApJS, 70, 1

Aryal, B., Paudel, S., Saurer, W. 2007, MNRAS, 379, 1011

Aryal, B., Saurer, W. 2006, MNRAS, 366, 438

Aryal, B., Saurer, W. 2005, A\&A, 432, 841

Bergh, V.D. 1959, Publ. David Dunlop Obs. 2, 145

Bergh, V.D. 1966, AJ, 71, 922

Binney J., Tremaine, S. 1987, Galactic Dynamics, Princeton Univ. press, Princeton, New Jersey

Brandner, W., Grebel, E.K., Chu, Y.-H. et al. 2000, AJ, 119, 292

Brunzendorf, J., Meusinger H. 1999, A\&AS, 139, 141

Capozziello, S., Lattanzi, A. 2006, Ap\&SS, 301, 1-4, 189

Corwin, H.G., de Vaucouleurs, A., de Vaucouleurs, G. 1985, Univ. Texas Monogr. Astron., 4, 1

Dreyer, J.L.E. 1895, MNRAS, 51, 185

Dreyer, J.L.E. 1908, MNRAS, 59, 105

Doroshkevich, A.G. 1973, ApJ, 14, L11

de Vaucouleurs, G., de Vaucouleurs, A., Corwin, et al. 1991, Third Reference Catalogue of Bright

Galaxies, Springer-Verlag, New York

Godlowski, W. 1993, MNRAS, 265, 874

Impey, C.D., Sprayberry, D., Irwin, M.J., Bothun, G.D. 1996, Astrophys. J. Supp., 105, 209 
Kodaira, K., Okamura, S., Ichikawa, S. 1990, Photometric Atlas of Northern Bright Galaxies, Univ. of Tokyo Press, Tokyo

Lauberts, A. 1982, ESO/Uppsala Survey of the ESO B Atlas, Garching bei Muenchen

Longo, M. J. 2007, preprint (astro-ph/0703325)

Land K., Slosar A., Lintott C. et al. 2008, MNRAS, 388, 1686

MacAlpine, G.M., Smith, S.B., Lewis, D.W. 1977a, ApJS, 34, 95

MacAlpine, G.M., Smith, S.B., Lewis, D.W. 1977b, ApJS, 35, 197

MacAlpine, G.M., Lewis, D.W., Smith, S.B. 1977c, ApJS, 35, 203

MacAlpine, G.M., Lewis, D.W. 1978, ApJS, 36, 587

MacAlpine, G.M., Williams, G.A. 1981, ApJS, 45, 113

Markarian, B.E. 1967, Astrofizika, 3, 24

Markarian, B.E., Lipovetskii, V.A., Stepanian, D.A. 1983, Astrofizika, 19, 29

Messier, C. 1784, Catalogue des nebuleuses et des amas d'etoiles, Connaissance des Temps

Nilson, P. 1973, Uppsala General Catalogue of Galaxies, Nova Acta Uppsala University, Ser. V:A, Vol.1

Nilson, P. 1974, Upps. Astron. Obs. Rep., 5

Oort, J.H. 1970a, Science, 170, 1363

Oort, J.H. 1970b, A\&A, 7, 405

Ozernoy, L.M. 1978, in: Longair M.S., Einasto J., eds., The Large Scale Structure of the Universe, Proc.

IAU Symp. No. 79. Reidel, Dordrecht, p. 427

Peebles, P.J.E. 1969, ApJ, 155, 393

Pasha, I.I. 1985, Sov. Astron. Lett., 11, 1

Shectman, S.A., Landy, S.D., Oemler, A. et al. 1996, ApJ, 470, 172

Struble, M.F., Rodd, H.J. 1999, ApJS, 125, 355

Sugai, H. \& Iye, M. 1995, MNRAS, 276, 327

Takase, et al. 1980-2000, Kiso Ultraviolet Galaxy Catalog, Publ. Nat. Astron. Obs. Japan

Thomasson, M., Donner, K. J., Sundelius, B. et al. 1989, A\&A, 211, 25

Vorontsov-Vel, Y.B.A., Krasnogorskaya, A.A., Arkhipova, V.P. 1962-74, Morphological Catalogue of Galaxies (Part I-V), Trudy Gosud. Astron. Inst. Shternberga

Zwicky, F., Wild, P., Karpowicz, M., Kowal, C.T. 1961-68, The Catalogue of Galaxies and Clusters of Galaxies, California Inst. of Tech., Pasadena 
Research in Astron. Astrophys. 2010 Vol.XX No.X, 000-000

http://www.raa-journal.org http://www.iop.org/journals/raa

\title{
Winding sense of galaxies around the Local Supercluster
}

\author{
B. Aryal ${ }^{1,2}$ \\ 1 Central Department of Physics, Tribhuvan University, Kirtipur, Kathmandu, Nepal \\ binil.aryal@uibk.ac.at \\ ${ }^{2}$ Institut für Astro- und Teilchenphysik, Universität Innsbruck, Technikerstraße 25, A-6020 \\ Innsbruck, Austria \\ Received [year] [month] [day]; accepted [year] [month] [day]
}

\begin{abstract}
We present an analysis of the winding sense (S and Z-shaped) of 1621 field galaxies that have radial velocity between $3000 \mathrm{~km} \mathrm{~s}^{-1}$ to $5000 \mathrm{~km} \mathrm{~s}^{-1}$. The preferred alignments of S- and Z-shaped galaxies are studied using chi-square, autocorrelation and the Fourier tests. We classified total galaxies into 32 subsamples and noticed a good agreement between the position angle (PA) distribution of S- and Z-shaped galaxies. The homogeneous distribution of the S- and Z-shaped galaxies is noticed for the late-type spirals ( $\mathrm{Sc}, \mathrm{Scd}, \mathrm{Sd}$ and $\mathrm{Sm}$ ) than that of the early-types ( $\mathrm{Sa}, \mathrm{Sab}, \mathrm{Sb}$ and $\mathrm{Sbc}$ ). A significant dominance of S-mode galaxies is noticed in the barred spirals. A random alignment is noticed in the PA-distribution of Z- and S-mode spirals. In addition, homogeneous distribution of the S- and Z-shaped galaxies is found to be invariant under the global expansion. The PAdistribution of the total S-mode galaxies is found to be random, whereas preferred alignment is noticed for the total Z-mode galaxies. It is found that the galactic planes of Z-mode galaxies tend to lie in the equatorial plane.
\end{abstract}

Key words: spiral galaxies - clusters: individual (Local Supercluster)

\section{INTRODUCTION}

Differential rotation in a galaxy's disc generate density waves in the disc, leading to spiral arms. According to gravitational theory, the spiral arms born as leading and subsequently transform to trailing modes. With the passage of time, the spiral pattern deteriorates gradually by the differential rotation of the equatorial plane of the galaxy, but the bar structure persists for a long time (Oort 1970a). This structure can again regenerate spiral pattern in the outer region. Thus, a close relation between the origin of the arms in the spirals and barred spirals can not be denied (Oort 1970b).

Land et al. (2008) studied the distribution of projected spin vectors of $\sim 37000$ spiral galaxies taken from Solon Dizital Sky Survey. They did not notice any evidence for overall preferred handedness of Universe. In a similar study, Longo (2007) found evidence for a preferred axis. Sugai \& Iye (1995) used statistics and studied the winding sense of galaxies (S- and Zpatterns) in 9825 spirals. No significant dominance from a random distribution is noticed. Aryal \& Saurer (2005) studied the spatial orientations of spin vectors of 4073 galaxies in the Local Supercluster. No preferred alignment is noticed for the total sample. These results hint that 
the distribution of angular momentum of galaxies is entirely random in two- (S- and Z-shaped) and three-dimensional (spin vector) analysis provided the database is rich.

In order to understand true structural modes (leading or trailing) of spiral galaxies, we need to know the direction of the spiral pattern (S- or Z-patterns), the approaching and receding sides and the near and far parts, since galaxies are commonly inclined in space to the line of the sight. The S and Z-patterns can be determined from the image of the galaxy. Similarly, the approaching and receding sides can be defined if spectroscopy data on rotation is available. The third one is fairly hard to established. For this, Pasha (1985) used 'tilt' criteria and studied the sense of winding of the arms in 132 spirals. He found 107 spirals to have trailing arm. Thomasson et al. (1989) studied theoretically and performed $N$-body simulations in order to understand the formation of spiral structures in retrograde galaxy encounters. Interestingly, they noticed the importance of halo mass. They concluded that the spirals having halos with masses larger than the disk mass exhibit leading pattern. Thus, the makeup of galactic haloes is important to cosmology in order to understand the evolution of galaxies.

By considering the group of transformations acting on the configuration space, Capozziello \& Lattanzi (2006) predicted that the progressive loss of inhomogeneity in the S- and Z-shaped galaxies might have some connection with the rotationally-supported (spirals, barred spirals) and randomized stellar systems (lenticulars, ellipticals).

The preferred alignments of galaxies can be an indicator of initial conditions when galaxies and clusters formed provided the angular momenta of galaxies have not been altered too much since their formation. A useful property of galaxies in clusters for which theories make different predictions is the angular momentum distribution. The 'Pancake model' by Doroshkevich (1973), the 'Hierarchy model' by Peebles (1969) and, the 'Primordial vorticity model' by Ozernoy (1978) predict different scenarios concerning the formation of large-scale structure. Thus, the study of galaxy orientation has the potential to yield important information regarding the formation and evolution of cosmic structures. In this work, we present an analysis of winding sense and preferred alignments of galaxies that have radial velocity (RV) $3000 \mathrm{~km}$

$\mathrm{s}^{-1}$ to $5000 \mathrm{~km} \mathrm{~s}^{-1}$. These are field galaxies. We intend to study the importance of winding sense in order to understand the true structural modes (i.e., leading and trailing arm) of the galaxy. We expect to study the following: (1) Are the distribution of S- and Z-shaped galaxies homogeneous in the field? (2) Is there any correlation between the preferred alignment and the winding sense of galaxies? (3) Does radial velocity dependence exist concerning winding sense of galaxies? and finally, (4) What can we say about the distribution of true structural modes (i.e., leading or trailing arm) of galaxies in the large scale structure?

This paper is organized as follows: in Sect. 2 we describe the method of data reduction. In Sect. 3 we give a brief account of the methods and the statistics used. Finally, a discussion of the statistical results and the conclusions are presented in Sects. 4 and 5.

\section{THE SAMPLE: DATA REDUCTION}

Eighteen catalogues were used for the data compilation. A list of the catalogues and their references are given in Table 1. The abbreviations given in the first column of Table 1 are as follows: NGC - New General Catalogue, UGC - Uppsala General Catalogue of Galaxies, ESO - ESO/Uppsala Survey of the ESO (B) Atlas, IC - Index Catalogue, MCG - Morphological Galaxy Catalogue, UGCA - Uppsala obs. General Catalogue, Addendum, CGCG - Catalogue of Galaxies and Clusters of Galaxies, KUG - Kiso Ultraviolet Galaxy Catalogue, MRK Markarian Galaxy Catalogue, MESSIER - Catalogue des nebuleuses et des amas d'etoiles, BCG - Brandner+Grebel+Chu Catalogue, LSBG - Low Surface Brightness Galaxies, SBS - Second Byurakan Survey, LCRS - Las Companas Red Shift Survey, DDO - David Dunlap Observatory Publications, IRAS - Infrared Astronomical Satellite, SGC - Southern Galaxy Catalogue and UM - University of Michigan: Curtis Schmidt-thin prism survey for extragalactic emission-line objects: List I-V. 


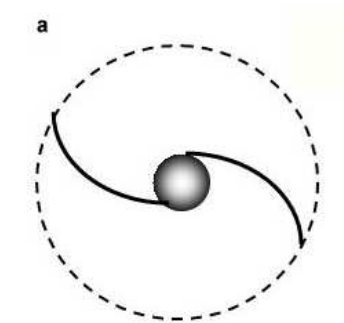

S-mode

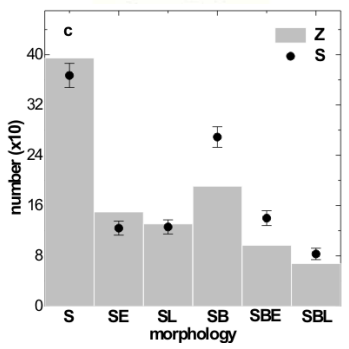

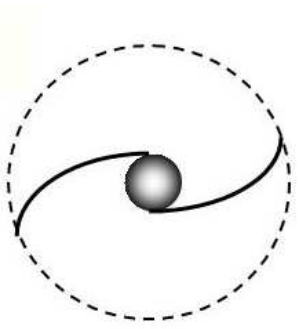

Z-mode

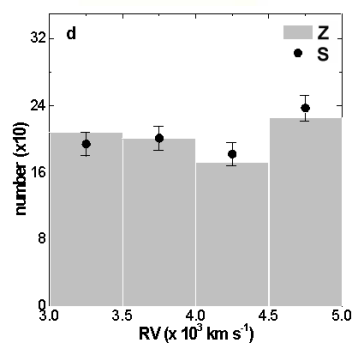

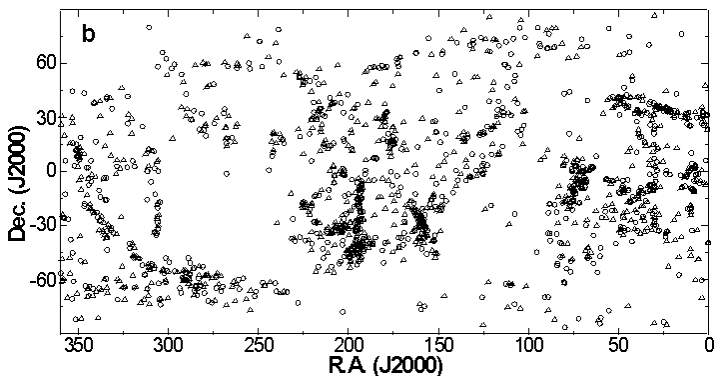
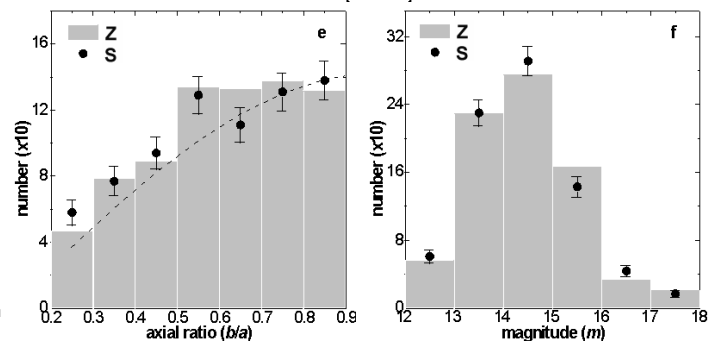

Fig. 1 (a) A sketch representing the winding sense ( $\mathrm{S}$ or $\mathrm{Z}$ ) of the galaxy. (b) Allsky distribution of Z-mode $(\triangle)$ and S-mode (o) galaxies that have RVs in the range $3000 \mathrm{~km} \mathrm{~s}^{-1}$ to $5000 \mathrm{~km} \mathrm{~s}^{-1}$. The morphology (c), radial velocity (d), axial ratio (e) and the magnitude (f) distribution of $\mathrm{Z}$ and $\mathrm{S}$-mode galaxies in our database. The statistical $\pm 1 \sigma$ error bars are shown for the S-mode $(\bullet)$ subsample. The dashed line (e) represents the expected distribution.

The NASA/IPAC extragalactic database (NED, http://nedwww.ipac.caltech.edu/) was used to compile these catalogues. The main editing process was as follows: first, galaxies having RVs in the range $3000 \mathrm{~km} \mathrm{~s}^{-1}$ to $5000 \mathrm{~km} \mathrm{~s}^{-1}$ were collected. We downloaded the image of all these galaxies from NED in FITS format. The second step was to compile the morphology of these galaxies from the catalog. A galaxy with doubtful morphology (eg., 'S?', 'S0' or 'Sa') is omitted. Finally, the position angles (PAs) of galaxies were added from the UGC, ESO, and Third Reference Catalogue of Bright Galaxies (de Vaucouleurs et al. 1991).

There were two clusters Abell $0426\left(\alpha(\mathrm{J} 2000)=03^{\mathrm{h}} 18^{\mathrm{m}} 36.4^{\mathrm{s}}, \delta(\mathrm{J} 2000)=+41^{\circ} 30^{\prime} 54^{\prime \prime}\right)$ and Abell $3627\left(\alpha(\mathrm{J} 2000)=16^{\mathrm{h}} 15^{\mathrm{m}} 32.8^{\mathrm{s}}, \delta(\mathrm{J} 2000)=-60^{\circ} 54^{\prime} 30^{\prime \prime}\right)$ in our region. These clusters have mean RVs $5366 \mathrm{~km} \mathrm{~s}^{-1}(75 \pm 5 \mathrm{Mpc})$ and $4881 \mathrm{~km} \mathrm{~s}^{-1}(63 \pm 4 \mathrm{Mpc})$, respectively (Abell, Corwin \& Olowin 1989, Struble \& Rodd 1999). We removed the galaxies belong to the cluster Abell 0426 using the catalog established by Brunzendorf \& Meusinger (1999). For the cluster Abell 3627 galaxies, we used Photometric Atlas of Northern Bright Galaxies (Kodaira, Okamura \& Ichikawa 1990) and Uppsala Galaxy Catalogue (Nilson 1973). There were 174 galaxies belongs to these clusters in our database. We remove these galaxies.

The RVs were compiled from Las Campanas Redshift Survey (Shectman 1996). The PAs and the diameters of galaxies were added from the Uppsala Galaxy Catalogue (Nilson 1973), Uppsala obs. General Catalogue, Addendum (Nilson 1974), Photometric Atlas of Northern Bright Galaxies (Kodaira, Okamura, \& Ichikawa 1990), ESO/Uppsala Survey of the European Southern Observatory (Lauberts 1982), Southern Galaxy Catalogue (Corwin et al. 1985) and Third Reference Catalogue of Bright Galaxies (de Vaucouleurs et al. 1991).

In the NED, 6493 galaxies having RVs $3000 \mathrm{~km} \mathrm{~s}^{-1}$ to $5000 \mathrm{~km} \mathrm{~s}^{-1}$ were listed until the cutoff date. Morphological information was given in the catalogues for $3276(50 \%)$ galaxies. We visually inspected all these galaxies using ALADIN2.5 software. 
Table 1 The list of catalogues used for the data compilation. The first column lists the abbreviation of the catalogue. The second column gives the total number of galaxies. The references are listed in the last column.

\begin{tabular}{lrl}
\hline Catalogue & N & References \\
\hline NGC & 623 Dreyer (1895, 1908) \\
UGC & 276 Nilson (1973) \\
ESO & 123 Lauberts (1982) \\
IC & 93 Dreyer (1895, 1908) \\
MCG & 88 Vorontsov-Vel'Yaminov et al. \\
& $\quad$ (1962-74) \\
UGCA & 84 Nilson (1974) \\
CGCG & 75 Zwicky et al. (1961-68) \\
KUG & 44 Takase (1980-2000) \\
MRK & 40 Markarian (1967) \\
MESSIER & 41 Messier (1784) \\
BCG & 28 Brandner et al. (2000) \\
LSBG & 23 Impey et al. (1996) \\
SBS & 17 Markarian et al. (1983) \\
LCRS & 16 Shectman et al. (1996) \\
DDO & 15 Bergh (1959, 1966) \\
IRAS & 15 Infrared Astronomical Satellite \\
& & (1983) \\
SGC & 10 Corwin et al. (1985) \\
UM & 10 MacAlpine et al. \\
& & (1977a,b,c; 1978; 1981) \\
\hline
\end{tabular}

The arm patterns (S- or Z-type) of the galaxies were studied visually by the author in order to maintain homogeneity. The contour maps of the galaxies were studied in order to identify their structural modes. For this, we used ALADIN2.5 software. The Z-mode is one whose outer tip points towards the anti-clockwise direction (see Fig. 1a). Similarly, the outer tip of S-mode directs in the clockwise direction. These two patterns are obviously the two dimensional projections of three dimensional galaxy. The re-examination of the S- and Z-modes using MIDAS software resulted the rejection of more than $17 \%$ of the objects. These rejected galaxies were nearly edge-on spiral and barred spiral galaxies. As expected, it was relatively easier to identify the structural modes of nearly face-on than that of nearly edge-on galaxies.

In this way, we compiled a database of 1621 galaxies showing either S- or Z-structural mode. There were 807 Z-mode and 821 S-mode galaxies in our database. All sky distribution of Zand S-mode galaxies is shown in Fig. 1b. The symbols "०" and " $\triangle$ " represent the positions of the S-mode and Z-mode galaxies, respectively. Several groups and aggregations of the galaxies can be seen in the figure.

The morphology, radial velocity, axial ratio and the magnitude distributions of S- and Zmode galaxies are shown in Figs. 1c,d,e,f. The spirals (47\%) dominate our database (Fig. 1c). However, a significant dominance of S-modes are noticed in the barred spirals whereas a weak dominance of Z-modes are found in the spirals. The population of galaxies in the RV distribution $\left(\Delta \mathrm{RV}=500 \mathrm{~km} \mathrm{~s}^{-1}\right)$ were nearly equal (Fig. 1d). The axial ratio distribution shows a good agreement with the expected cosine curve in the limit $0.2<b / a \leq 0.9$ (Fig. 1e). The values of absolute magnitude lie between 13.0 and 16.0 for $82 \%$ galaxies in our database (Fig. 1f).

We classified the database into 32 subsamples for both the $\mathrm{S}$ - and Z-modes on the basis of the morphology, radial velocity, area and the group of the galaxies. The galaxies with doubtful morphology are omitted in the spiral and barred spiral subsamples. The total number of earlyand late-type spirals or barred spirals is much less than that of the total spiral or total barred spirals. It is because of the fact that the galaxy with incomplete morphology, say, simply ' $\mathrm{S}$ ' or 'SB' can not be included in the subsamples. In other words, the galaxy with morphology Sa, 
$\mathrm{Sab}, \mathrm{Sb}, \mathrm{Sbc}$ are included in the early spirals whereas the galaxies with morphology Sc, Scd, Sd and Sdm are classified as late type spirals. The galaxies having morphology other than Sa, Sab, $\mathrm{Sb}, \mathrm{Sbc}, \mathrm{Sc}, \mathrm{Scd}, \mathrm{Sd}$ and Sm can not be included in the early and late subsamples. A statistical study of these subsamples are given in Table 1 and discussed in Sect. 4.1.

\section{METHOD OF ANALYSIS}

Basic statistics is used to study the dominance of Z- or S-mode galaxies. At first, morphology and RV dependence are studied. Secondly, sky is divided into 16 equal parts in order to observe deviation from the homogeneity. Several galaxy groups are identified in the all-sky map where the structural dominance are noticed. Finally, we study the dominance of Z- or S-mode galaxies in these groups.

We assume isotropic distribution as a theoretical reference and studied the equatorial PAdistribution in the total sample and subsamples. In order to measure the deviation from isotropic distribution we have carried out three statistical tests: chi-square, auto-correlation and the Fourier.

We set the chi-square probability $\mathrm{P}\left(>\chi^{2}\right)=0.050$ as the critical value to discriminate isotropy from anisotropy, this corresponds to a deviation from isotropy at the $2 \sigma$ level (Godlowski 1993). Auto correlation test takes account the correlation between the number of galaxies in adjoining angular bins. We expect, auto correlation coefficient $\mathrm{C} \rightarrow 0$ for an isotropic distribution. The critical limit is the standard deviation of the correlation coefficient C.

If the deviation from isotropy is only slowly varying with angles (in our case: PA) the Fourier test can be applied (Godlowski 1993). A method of expanding a function by expressing it as an infinite series of periodic functions (sine and cosine) is called Fourier series. Let $N$ denote the total number of solutions for galaxies in the sample, $N_{k}$ the number of solutions in the $\mathrm{k}^{\text {th }}$ bin, $N_{0}$ the mean number of solutions per bin, and $N_{0 k}$ the expected number of solutions in the $\mathrm{k}^{\text {th }}$ bin. Then the Fourier series is given by (taking first order Fourier mode),

$$
N_{k}=N_{k}\left(1+\Delta_{11} \cos 2 \beta_{k}+\Delta_{21} \sin 2 \beta_{k}+\ldots \ldots\right)
$$

Here the angle $\beta_{k}$ represents the polar angle in the $\mathrm{k}^{t h}$ bin. The Fourier coefficients $\Delta_{11}$ and $\Delta_{21}$ are the parameters of the distributions. We obtain the following expressions for the Fourier coefficients $\Delta_{11}$ and $\Delta_{21}$,

$$
\begin{aligned}
& \Delta_{11}=\sum\left(N_{k}-N_{0 k}\right) \cos 2 \beta_{k} / \sum N_{0 k} \cos ^{2} 2 \beta_{k} \\
& \Delta_{21}=\sum\left(N_{k}-N_{0 k}\right) \sin 2 \beta_{k} / \sum N_{0 k} \sin ^{2} 2 \beta_{k}
\end{aligned}
$$

The standard deviations $\left(\sigma\left(\Delta_{11}\right)\right)$ and $\left(\sigma\left(\Delta_{21}\right)\right)$ can be estimated using the expressions,

$$
\begin{aligned}
& \sigma\left(\Delta_{11}\right)=\left(\sum N_{0 k} \cos ^{2} 2 \beta_{k}\right)^{-1 / 2} \\
& \sigma\left(\Delta_{21}\right)=\left(\sum N_{0 k} \sin ^{2} 2 \beta_{k}\right)^{-1 / 2}
\end{aligned}
$$

The probability that the amplitude

$$
\Delta_{1}=\left(\Delta_{11}^{2}+\Delta_{21}^{2}\right)^{1 / 2}
$$

greater than a certain chosen value is given by the formula

$$
P\left(>\Delta_{1}\right)=\exp \left(-n N_{0} \Delta_{1}^{2} / 4\right)
$$

with standard deviation

$$
\sigma\left(\Delta_{1}\right)=\left(2 / n N_{0}\right)^{1 / 2}
$$

The Fourier coefficient $\Delta_{11}$ gives the direction of departure from isotropy. The first order Fourier probability function $P\left(>\Delta_{1}\right)$ estimates whether (smaller value of $P\left(>\Delta_{1}\right)$ or not (higher value of $P\left(>\Delta_{1}\right)$ a pronounced preferred orientation occurs in the sample. 


\section{RESULTS}

First we present the statistical result concerning the distribution of Z- and S-mode galaxies in the total sample and subsamples. Second, we study the distribution of Z- and S-mode galaxies in the unit area of the sky and in the groups. Then, the equatorial PA-distribution of galaxies in the total sample and subsamples are discussed. At the end, a general discussion and a comparison with the previous results will be presented.

\subsection{Distribution of $\mathrm{Z}$ and $\mathrm{S}$ mode galaxies}

A statistical comparison between the total sample and subsamples of the Z- and S-modes of galaxies is given in Table 2. Fig. 2 shows this comparison in the histogram. The $\Delta(\%)$ in Table 1 and Fig. 2 represent the percentage difference between the number Z- and S-mode galaxies. We studied the standard deviation of the major diameters $(a)$ of galaxies in the total sample and subsamples for both the Z- and S-modes. In Table $2, \Delta(a$ sde $)$ represents the difference between the standard deviation of the major diameters of Z- and S-mode galaxies.

An insignificant difference $(0.4 \% \pm 0.2 \%)$ between the total number of Z- and S-mode galaxies are found (Table 2). The difference between the standard deviation of the major diameters $(\Delta$ (a sde $))$ of the Z- and S-mode galaxies is found less than 0.019 (eighth column, Table 2). Interestingly, the sum of the major diameters of total Z- and S-mode galaxies coincide. This result suggests the homogeneous distribution of Z- and S-mode field galaxies that have RV in the range $3000 \mathrm{~km} \mathrm{~s}^{-1}$ to $4000 \mathrm{~km} \mathrm{~s}^{-1}$.

In Fig. 2, the slanting-line (grey-shaded) region corresponds to the region showing $\leq 10 \%$ (5\%) $\Delta$ value. Almost all subsamples lie in this region, suggesting the homogeneous distribution of Z- and S-mode galaxies within 10\% error limit. Now, we present the distribution Z- and Smode galaxies in the subsamples classified according as their morphology, RVs, area and the groups below.

\subsubsection{Morphology}

In the spirals, Z-mode galaxies are found 3.7\% $( \pm 1.8 \%)$ more than that of S-mode. The homogeneous distribution of Z- and S-modes is found for the late-type spirals (Sc, Scd, Sd and Sm) than that of early-type (Sa, Sab, Sb and Sbc): $\Delta$ value turned out to be $9.5 \%( \pm 4.8 \%)$ and $1.8 \%( \pm 1.0 \%)$ for early- and late-types (Table 2$)$. Thus, no preferred winding pattern is noticed in the late-type spirals than that of early-types.

A significant dominance of S-mode galaxies are noticed $(17 \% \pm 8.5 \%)$ in spiral barred galaxies. The $\Delta$ value is found $>9 \%$ for both early- (SBa, SBab, SBb and SBbc) and late-type (SBc, $\mathrm{SBcd}, \mathrm{SBd}, \mathrm{SBm}$ ) barred spirals. Similar result (i.e., $\Delta>8 \%$ ) is found for the irregulars and the morphologically unidentified galaxies.

A similarity is noticed between the late-type spirals and barred spirals: the $\Delta$ value for both the late-types are found to be less than that of early-types (see Table 2).

The difference between the standard deviation of the major diameters $(\Delta(a$ sde $))$ for Sand Z-mode galaxies is found less than 0.050 arc minute for the total sample, spirals and the late-type spirals (eighth column, Table 1). These samples showed $\Delta$ value $<5 \%$ (grey-shaded region, Fig. 2a). Thus, we noticed a good correlation between the $\Delta(\%)$ and $\Delta(a$ sde $)$ value.

The difference between the sum of the major diameters (in percentage) are found greater than $10 \%$ for the barred spirals and early-type barred spirals. Interestingly, these two subsamples showed $\Delta$ value greater than $15 \%$ (Fig. 2a). Thus, inhomogeniety in the distribution of S- and Z-mode galaxies is noticed for barred spirals. 

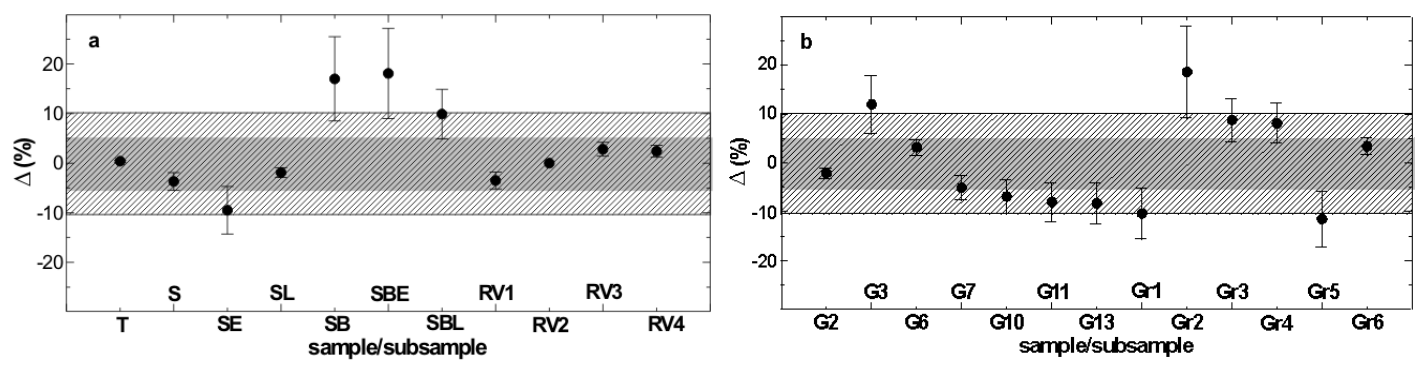

Fig. 2 The basic statistics of the $\mathrm{Z}$ and S-mode of galaxies in the total sample and subsamples. The full form of the abbreviations (X-axis) are given in Table 2 (first column). $\Delta(\%)=S-Z$, where $S$ and $Z$ represent the number of S- and Z-mode galaxies, respectively. The statistical error bars $\sigma(\%)$ shown in the figure are calculated as: $\sigma(\%)$ $=\sigma /(\sqrt{S}+\sqrt{Z}) \times 100$, where $\sigma=(\sqrt{S}-\sqrt{Z})$. The grey-shaded and the slanting-line region represent the $\leq \pm 5 \%$ and $\leq \pm 10 \% \Delta$ value, respectively.

\subsubsection{Radial velocity}

A very good correlation between the number of S- and Z-mode can be seen in the RV classifications (Fig. 1d). All 4 subsamples show the $\Delta$ and $\Delta(a$ sde) values less than $5 \%$ and 0.050 , respectively (Table 2). In addition, $\Delta(a)$ is found to be $<5 \%$. This result is important in the sense that the statistics in these subsamples is rich (number of galaxies $>170$ ) enough. Thus, we could not observe preference structural modes ( $\mathrm{S}$ or $\mathrm{Z}$ ) in the low and high RV galaxies in our database.

A difference is noticed: dominance of Z- and S- modes, respectively in low (RV1) and high (RV3, RV4) RV subsamples. However, this dominance is not significant (i.e., $\Delta<5 \%$ ). An equal number of S- and Z-mode galaxies are found in the subsample RV2 $\left(3500<\mathrm{RV}\left(\mathrm{km} \mathrm{s}^{-1}\right) \leq\right.$ 4000 ) (Table 1). In order to check the binning effect, we further classify the total galaxies in 6 $\left(\Delta \mathrm{RV}=333 \mathrm{~km} \mathrm{~s}^{-1}\right)$ and 8 bins $\left(\Delta \mathrm{RV}=250 \mathrm{~km} \mathrm{~s}^{-1}\right)$ and study the statistics. No significant dominance of either S- and Z-modes are noticed.

Thus, it is found that the homogeneous distribution of S- and Z-mode galaxies remain invariant with the global expansion (i.e, expansion of the Universe). We further discuss this result below.

\subsubsection{Area}

We study the distribution of S- and Z-mode galaxies by dividing the sky into 16 equal parts (Fig. 3a). The area of the grid $(\mathrm{G})$ is $90^{\circ} \times 45^{\circ}(\mathrm{RA} \times \mathrm{Dec})$. The area distribution of S- and Z-mode galaxies are plotted, that can be seen in Fig. 3a'. The statistical parameters are given in Table 2.

A significant dominance $(>2 \sigma)$ of S-mode is noticed in grid 3 (RA: $0^{\circ}$ to $90^{\circ}$, Dec: $0^{\circ}$ to $45^{\circ}$ (J2000)) (Fig. 3a, a'). An elongated group of galaxies can be seen in this grid. In this grid, $\Delta, \Delta(a$ sde) and $\Delta(a) \%$ are found to be $12 \% \pm 6 \%, 0.076$ and $9.3 \%$, respectively. These figures suggest that the distribution of S- and Z-mode galaxies in G3 is not homogeneous. Probably, this is due to the apparent subgroupings or subclusterings of the galaxies.

The S-mode galaxies dominate in the grids G8 and G9 (Fig. 3a'). However, the statistics is poor $(<40)$ in these grids (Table 2$)$. In addition, no groupings or subclustering are noticed.

A dominance $(\sim 1.5 \sigma)$ of Z-mode is noticed in G10 (RA: $180^{\circ}$ to $270^{\circ}$, Dec: $-45^{\circ}$ to $0^{\circ}$ (J2000)) and G13 (RA: $270^{\circ}$ to $360^{\circ}$, Dec: $-90^{\circ}$ to $-45^{\circ}$ (J2000)) (Fig. 3a,a'). In both the grids, a large aggregation of the galaxies can be seen. A subcluster-like aggregation can be seen in 
Table 2 Statistics of leading (column 3) and trailing arm (column 4) galaxies in the total sample and subsamples. The fifth and sixth column give the numeral and percentage difference $(\Delta=S-Z)$ between the S- $(S)$ and the Z- $(Z)$ modes. The next two columns give the error: $\sigma=(\sqrt{S}-\sqrt{Z})$ and $\sigma(\%)=\sigma /(\sqrt{S}+\sqrt{Z}) \times 100$. The eighth column gives the difference between the standard deviation (in arcmin) of the major diameters $(a)$ of the S- and Z-modes galaxies $(\Delta($ a sde $))$. The difference between the sum of the major diameters $(\Delta(a) \%)$ are listed in the last column. The sample/subsample and their abbreviations are given in first two columns.

\begin{tabular}{|c|c|c|c|c|c|c|c|c|}
\hline sample/subsample & symbol & $\mathrm{Z}$ & $\mathrm{S}$ & $\Delta$ & $\Delta(\%)$ & $\sigma(\%)$ & $\Delta(a$ sde $)$ & $\Delta(a)(\%)$ \\
\hline Total & $\mathrm{T}$ & 814 & 807 & -7 & -0.4 & -0.2 & 0.019 & 0.0 \\
\hline Spiral & S & 395 & 367 & -28 & -3.7 & -1.8 & 0.031 & 3.0 \\
\hline Spiral (early-type) & SE & 150 & 124 & -26 & -9.5 & -4.8 & 0.058 & 8.1 \\
\hline Spiral (late-type) & SL & 131 & 126 & -5 & -1.9 & -1.0 & 0.031 & 0.3 \\
\hline Barred Spiral & SB & 191 & 269 & 78 & 17.0 & 8.5 & 0.062 & 15.2 \\
\hline Barred Spiral (early-type) & SBE & 97 & 140 & 43 & 18.1 & 9.1 & 0.091 & 14.6 \\
\hline Barred Spiral (late-type) & SBL & 68 & 83 & 15 & 9.9 & 5.0 & 0.066 & 9.2 \\
\hline $3000<\mathrm{RV}\left(\mathrm{km} \mathrm{s}^{-1}\right) \leq 3500$ & RV1 & 208 & 194 & -14 & -3.5 & -1.7 & 0.046 & 2.6 \\
\hline $3500<\mathrm{RV}\left(\mathrm{km} \mathrm{s}^{-1}\right) \leq 4000$ & RV2 & 201 & 201 & 0 & 0.0 & 0.0 & 0.031 & 3.1 \\
\hline $4000<\mathrm{RV}\left(\mathrm{km} \mathrm{s}^{-1}\right) \leq 4500$ & RV3 & 172 & 182 & 10 & 2.8 & 1.4 & 0.034 & 0.6 \\
\hline $4500<\mathrm{RV}\left(\mathrm{km} \mathrm{s}^{-1}\right) \leq 5000$ & RV4 & 226 & 237 & 11 & 2.4 & 1.2 & 0.041 & 1.0 \\
\hline Grid 1 & G1 & 20 & 21 & 1 & 2.4 & 1.2 & 0.068 & 6.8 \\
\hline Grid 2 & G2 & 121 & 116 & -5 & -2.1 & -1.1 & 0.014 & 1.6 \\
\hline Grid 3 & G3 & 88 & 112 & 24 & 12.0 & 6.0 & 0.076 & 9.3 \\
\hline Grid 4 & G4 & 12 & 14 & 2 & 7.7 & 3.9 & 0.647 & 9.7 \\
\hline Grid 5 & G5 & 11 & 8 & $-\overline{3}$ & -15.8 & -7.9 & 0.042 & 22.3 \\
\hline Grid 6 & G6 & 75 & 80 & 5 & 3.2 & 1.6 & 0.081 & 4.5 \\
\hline Grid 7 & G7 & 62 & 56 & -6 & -5.1 & -2.5 & 0.095 & 8.9 \\
\hline Grid 8 & G8 & 22 & 33 & 11 & 20.0 & 10.1 & 0.073 & 12.9 \\
\hline Grid 9 & G9 & 20 & 31 & 11 & 21.6 & 10.9 & 0.028 & 18.1 \\
\hline Grid 10 & G10 & 124 & 108 & -16 & -6.9 & -3.5 & 0.004 & 5.4 \\
\hline Grid 11 & G11 & 61 & 52 & -9 & -8.0 & -4.0 & 0.025 & 6.2 \\
\hline Grid 12 & G12 & 20 & 20 & 0 & 0.0 & 0.0 & 0.409 & 7.4 \\
\hline Grid 13 & G13 & 78 & 66 & -12 & -8.3 & -4.2 & 0.039 & 2.9 \\
\hline Grid 14 & G14 & 37 & 44 & 7 & 8.6 & 4.3 & 0.356 & 10.3 \\
\hline Grid 15 & G15 & 47 & 44 & -3 & -3.3 & -1.6 & 0.050 & 5.2 \\
\hline Grid 16 & G16 & 9 & 9 & 0 & 0.0 & 0.0 & 0.191 & 8.6 \\
\hline Group 1 & Gr1 & 37 & 30 & -7 & -10.4 & -5.2 & 0.032 & 7.1 \\
\hline Group 2 & Gr2 & 48 & 70 & 22 & 18.6 & 9.4 & 0.097 & 12.6 \\
\hline Group 3 & Gr3 & 31 & 37 & 6 & 8.8 & 4.4 & 0.027 & 4.3 \\
\hline Group 4 & Gr4 & 34 & 40 & 6 & 8.1 & 4.1 & 0.031 & 3.9 \\
\hline Group 5 & Gr5 & 107 & 85 & -22 & -11.5 & -5.7 & 0.089 & 11.2 \\
\hline Group 6 & Gr6 & 42 & 45 & 3 & 3.4 & 1.7 & 0.024 & 1.6 \\
\hline
\end{tabular}

G10. An elongated structure can be seen in G13. In both the grids, $\Delta$ value is found to be greater than $5 \%$ (Table 2).

No dominance of either S- and Z-mode galaxies is noticed in the groups G1, G2, G4, G5, G6, G7, G11, G12, G14, G15 and G16. Thus, homogeneous distribution of S- and Z-mode galaxies is found intact in $\sim 80 \%$ area of the sky. We suspect that the groupings or subclusterings of the galaxies lead the preference structural modes (S or Z) in G3, G10 and G13.

\subsubsection{Galaxy groups}

In all-sky map, several groups of galaxies can be seen (Fig. 3a). It is interesting to study the distribution of structural modes ( $\mathrm{S}$ or $\mathrm{Z}$ ) of galaxies in these groups. For this, we systematically searched for the groups fulfilling following selection criteria: (a) major diameter $>30^{\circ}$, (b) 

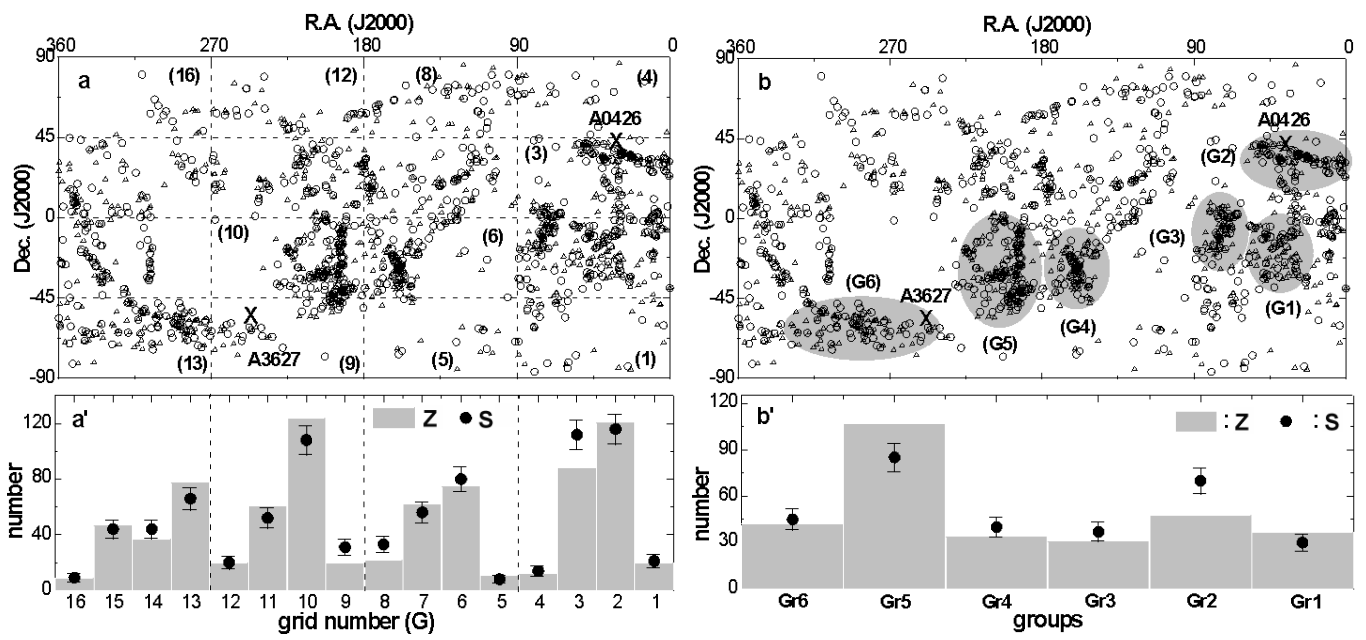

Fig. 3 (a) All sky distribution of Z-mode (hollow circle) and S-mode (hollow triangle) galaxies in 16 area grids. (a') The histogram showing the distribution of the Z- and S-mode galaxies in the grids G1 to G16. (b) Six groups of the galaxies, represented by the grey-shaded region. (b') The distribution of Z- and S-modes in 6 groups. The statistical error bar $\pm 1 \sigma$ is shown. The positions of the clusters Abell 0426 and Abell 3627 are shown by the symbol " $\times$ " $(\mathrm{a}, \mathrm{b})$.

cutoff diameter $<2$ times the background galaxies, (c) number of galaxies $>50$. We found 6 groups fulfilling these criteria (Fig. 3b). All 6 groups (Gr) are inspected carefully. In 3 groups (Gr2, Gr5 and Gr6), subgroups can be seen. The number of galaxies in the groups Gr2 and Gr5 are found more than 100 .

The clusters Abell 0426 and Abell 3627 are located close to the groups Gr2 and Gr6. The symbol " $\times$ " represents the position of the cluster center in Fig. 3b. The mean radial velocities of these clusters are $5366 \mathrm{~km} \mathrm{~s}^{-1}$ and $4881 \mathrm{~km} \mathrm{~s}^{-1}$, respectively. However, we have removed the member galaxies of these clusters from our database.

A significant dominance $(>2 \sigma)$ of S-mode galaxies is noticed in the group Gr2 (Fig. 3b,b'). The $\Delta, \Delta(a$ sde $)$ and $\Delta(a) \%$ values are found to be $18.6 \%( \pm 9.4 \%), 0.097$ and $12.6 \%$, respectively (Table 2). We suspect that the galaxies in this group is under the influence of the cluster Abell 0426, due to which apparent subclustering of the galaxies is seen.

The galaxies in Gr5 shows an opposite preference: a significant dominance of the Z-mode galaxies $(>2 \sigma)$ (Fig. 3b,b'). In this group, $\Delta, \Delta($ a sde) and $\Delta(a) \%$ are found to be $11.5 \% \pm$ $5.7 \%, 0.089$ and $11.2 \%$, suggesting inhomogeneous distribution of structural modes (Table 2).

No humps or dips can be seen in the groups Gr1, Gr3, Gr4 and Gr6 (Fig. 3.2b,b'). Thus, the distribution of S- and Z-mode galaxies in these groups are found to be homogeneous. The number of galaxies in these groups are less than 100 .

In the group 6, we could not notice the influence of the cluster Abell 3627. This might be due to the off location of the cluster center from the group center.

\subsection{Anisotropy in the position angle distribution}

We study the equatorial position angle (PA) distribution of S- and Z-mode galaxies in the total sample and subsamples. A spatially isotropic distribution is assumed in order to examine non-random effects in the PA-distribution. In order to discriminate the deviation from the randomness, we use three statistical tests: chi-square, auto correlation and the Fourier. The 


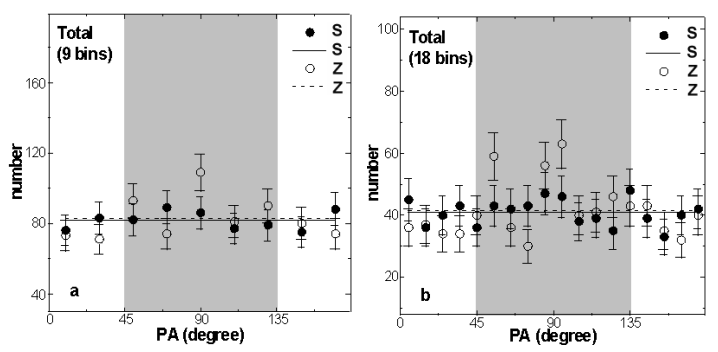

Fig. 4 The equatorial position angle (PA) distribution of total Z- and S-mode galaxies plotted in 9 (a) and 18 (b) bins. The solid and the dashed line represent the expected isotropic distribution for S- and Z-mode galaxies, respectively. The observed counts with statistical $\pm 1 \sigma$ error bars are shown. $\mathrm{PA}=90^{\circ} \pm 45^{\circ}$ (grey-shaded region) corresponds to the galactic rotation axes tend to be oriented perpendicular with respect to the equatorial plane.

bin size was chosen to be $20^{\circ}$ (9 bins) in all these tests. The statistically poor bins (number of solution $<5)$ are omitted in the analysis. The conditions for anisotropy are the following: the chi-square probability $\mathrm{P}\left(>\chi^{2}\right)<0.050$, correlation coefficient $C / \sigma(C)>1$, first order Fourier coefficient $\Delta_{11} / \sigma\left(\Delta_{11}\right)>1$ and the first order Fourier probability $\mathrm{P}\left(>\Delta_{1}\right)<0.150$ as used by Godlowski (1993). Table $\mathbf{3}$ lists the statistical parameters for the total samples and subsamples.

In the Fourier test, $\Delta_{11}<0$ (i.e., negative) indicates an excess of galaxies with the galactic planes parallel to the equatorial plane. In other words, a negative $\Delta_{11}$ suggests that the rotation axis of galaxies tend to be oriented perpendicular with respect to the equatorial plane. Similarly, $\Delta_{11}>0$ (i.e., positive) indicates that the rotation axis of galaxies tend to lie in the equatorial plane.

In the histograms (see Figs. 4-7), a hump at $90^{\circ} \pm 45^{\circ}$ (grey-shaded region) suggests that the galactic planes of galaxies tend to lie in the equatorial plane. In other words, the rotation axes of galaxies tend to be oriented perpendicular with respect to the equatorial plane when there is excess number of solutions in the grey-shaded region in the histogram.

All three statistical tests show isotropy in the total S-mode galaxies. Thus, no preferred alignment is noticed for the total S-mode galaxies (solid circles in Fig. 4a). Interestingly, all three statistical tests show anisotropy in the total Z-mode galaxies. The chi-square and Fourier probabilities $\left(\mathrm{P}\left(>\chi^{2}\right), \mathrm{P}\left(>\Delta_{1}\right)\right)$ are found $1.5 \%(<5 \%$ limit $)$ and $8.5 \%(<15 \%$ limit $)$, respectively (Table 2). The auto correlation coefficient $(\mathrm{C} / \mathrm{C}(\sigma))$ turned $-3.2(>>1)$. The $\Delta_{11} / \sigma\left(\Delta_{11}\right)$ value is found to be negative at $\sim 2 \sigma$ level, suggesting that the rotation axes of Z-mode galaxies tend to be oriented perpendicular the equatorial plane. Three humps at $50^{\circ}(>1.5 \sigma), 90^{\circ}(>2 \sigma)$ and $130^{\circ}(1.5 \sigma)$ support this result (Fig. 4a). We checked the biasness in the results due to bin size by increasing and decreasing the number of bins. A similar statistical result is found for both structural modes. Fig. 4b shows the PA-distribution histogram for the total sample in 18 bins. The Z-mode galaxies show three significant humps in the grey-shaded region, supporting the results mentioned above.

Thus, we conclude isotropy for S-mode whereas anisotropy for Z-mode galaxies in the total sample.

\subsubsection{Morphology}

In the spirals, the chi-square and auto correlation tests show isotropy for both the S- and Zmodes. The first order Fourier probability is found greater than 35\%, suggesting no preferred alignment. However, the $\Delta_{11}$ value exceeds $1 \sigma$ limit $(-1.2 \sigma)$ in the S-mode spirals. A hump at 
Table 3 Statistics of the PA-distribution of galaxies in the total sample and subsamples (first column). The second, third, fourth and fifth columns give the chi-square probability $\left(\mathrm{P}\left(>\chi^{2}\right)\right)$, correlation coefficient $(\mathrm{C} / \mathrm{C}(\sigma))$, first order Fourier coefficient $\left(\Delta_{11} / \sigma\left(\Delta_{11}\right)\right)$, and first order Fourier probability $\mathrm{P}\left(>\Delta_{1}\right)$, respectively. The last four columns repeats the previous columns.

\begin{tabular}{lcccccccc}
\hline sample & \multicolumn{3}{c}{ S-mode } & \multicolumn{7}{c}{ Z-mode } \\
& $\mathrm{P}\left(>\chi^{2}\right)$ & $\mathrm{C} / \mathrm{C}(\sigma)$ & $\Delta_{11} / \sigma\left(\Delta_{11}\right)$ & $\mathrm{P}\left(>\Delta_{1}\right)$ & $\mathrm{P}\left(>\chi^{2}\right)$ & $\mathrm{C} / \mathrm{C}(\sigma)$ & $\Delta_{11} / \sigma\left(\Delta_{11}\right)$ & $\mathrm{P}\left(>\Delta_{1}\right)$ \\
\hline total & 0.666 & +0.0 & -0.9 & 0.381 & 0.015 & -3.2 & -1.9 & 0.085 \\
S & 0.511 & -0.7 & -1.2 & 0.434 & 0.225 & +0.4 & +0.8 & 0.383 \\
SE & 0.973 & +0.1 & -0.9 & 0.569 & 0.031 & +2.0 & +2.8 & 0.015 \\
SL & 0.234 & +0.5 & +0.8 & 0.209 & 0.460 & -0.1 & -0.5 & 0.345 \\
SB & 0.729 & +0.3 & +1.0 & 0.454 & 0.285 & -1.0 & -0.2 & 0.497 \\
SBE & 0.739 & +0.1 & -0.5 & 0.566 & 0.230 & -0.7 & +0.1 & 0.521 \\
SBL & 0.043 & +1.8 & +1.7 & 0.046 & 0.620 & -0.9 & -0.2 & 0.872 \\
RV1 & 0.910 & +0.3 & +0.8 & 0.362 & 0.369 & -0.9 & -0.6 & 0.285 \\
RV2 & 0.790 & +0.3 & -1.0 & 0.496 & 0.925 & -0.4 & -0.2 & 0.887 \\
RV3 & 0.050 & +1.6 & -1.5 & 0.083 & 0.033 & -1.8 & -2.3 & 0.046 \\
RV4 & 0.043 & -2.3 & -1.5 & 0.116 & 0.636 & +0.2 & -0.7 & 0.692 \\
Gr2 & 0.455 & +0.6 & +0.8 & 0.861 & 0.033 & -1.8 & +1.7 & 0.116 \\
Gr5 & 0.033 & -1.4 & -2.0 & 0.085 & 0.516 & +0.4 & -0.4 & 0.548 \\
\hline
\end{tabular}
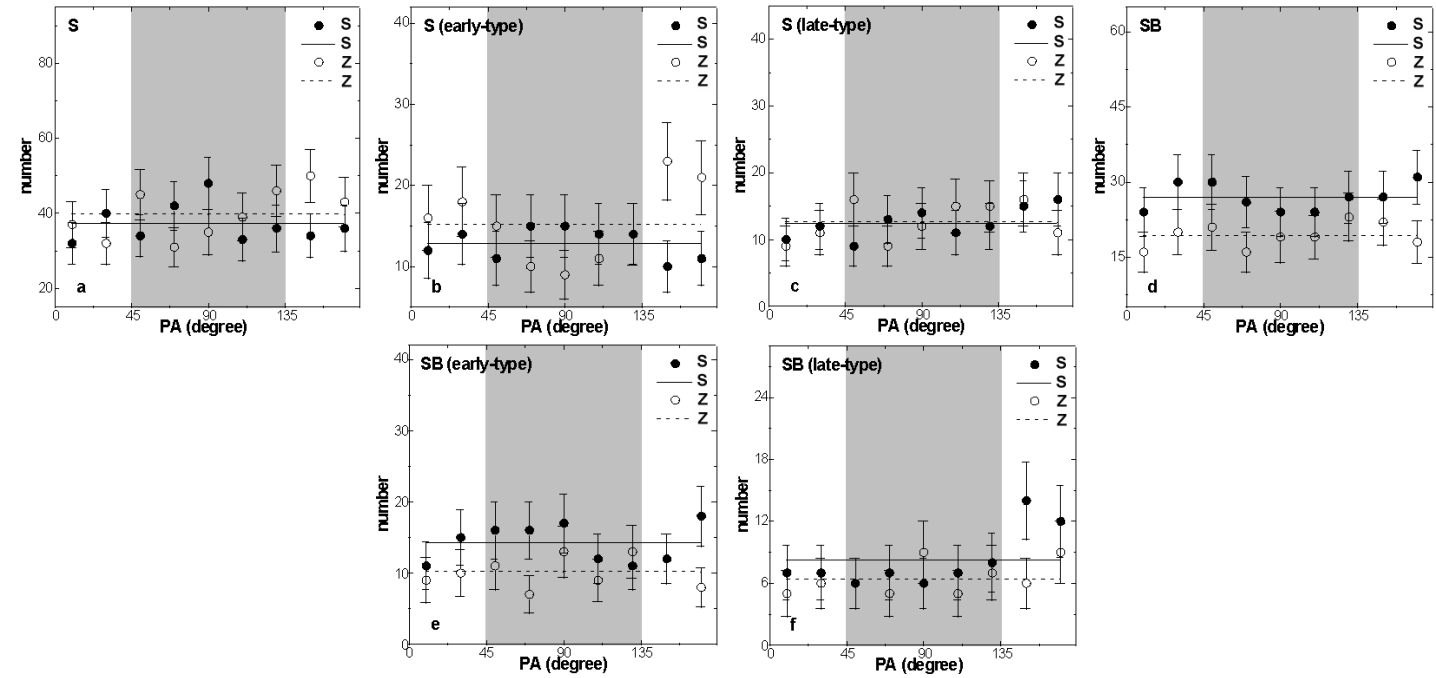

Fig. 5 The equatorial PA-distribution of Z- and S-mode galaxies in the spirals (a), early-type spirals (b), late-type spirals (c), barred spirals (d), early-type barred spirals (e) and late-type barred spirals (f). The symbols, error bars, dashed lines and the explanations are analogous to Fig. 4.

$90^{\circ}$ is not enough to turn the $\Delta_{11} / \sigma\left(\Delta_{11}\right)>1.5$ (Fig. 5a). Similarly, a hump at $150^{\circ}$ is not enough to make the $\Delta_{11} / \sigma\left(\Delta_{11}\right)>1.5$ in the Z-mode spirals. Hence, the preferred alignment is not profounded in both the S- and Z-mode spirals. Thus, we conclude a random orientation of S- and Z-mode spirals. 

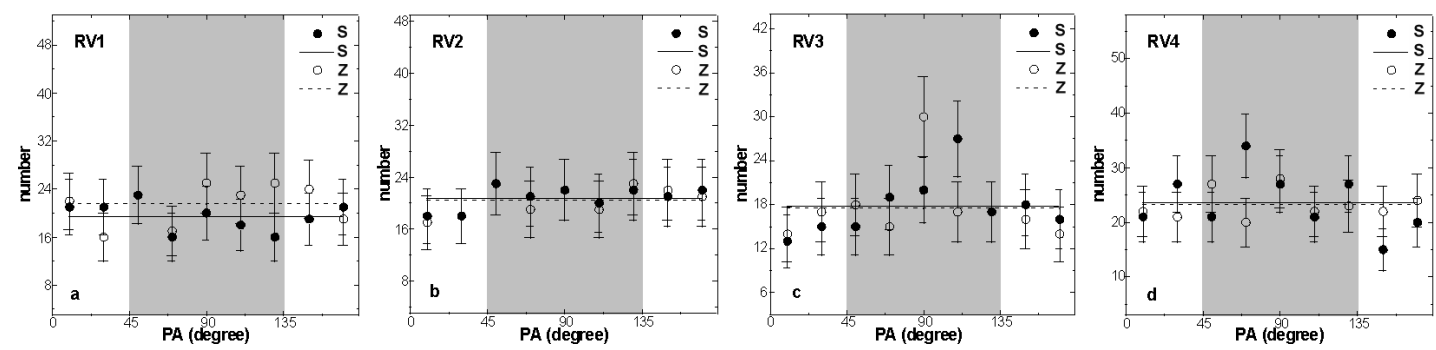

Fig. 6 The equatorial PA-distribution of Z- and S-mode galaxies in RV1 (a), RV2 (b), RV3 (c) and RV4 (d). The abbreviations are listed in Table 1. The symbols, error bars, dashed lines and the explanations are analogous to Fig. 4.

Early- and late-type S-mode spirals show isotropy in all three statistical tests (Table 3). No humps and the dips are seen in the histograms (solid circles in Fig. 5b,c). Thus, the Smode spirals show a random alignment in the PA-distribution. In the subsample SE, all three statistical tests show anisotropy (Table 2). Two significant humps at $>150^{\circ}$ cause the first order Fourier coefficient $\left(\Delta_{11}\right)>+2.5 \sigma$ (hollow circle in Fig. $5 \mathrm{~b}$ ). Thus, a preferred alignment is noticed in the early-type Z-mode spirals: the galactic rotation axes tend to lie in the equatorial plane. The late-type Z-mode spirals show a random alignment.

The spiral barred galaxies show a random alignment in both the S- and Z-modes. In Fig. $5 \mathrm{~d}$, no deviation from the expected distribution can be seen. All three statistical tests support this result (Table 2). A similar result is found for the early-type SB galaxies in both structural modes (Table 3, Fig. 5e).

The $\mathrm{P}\left(>\chi^{2}\right)$ and $\mathrm{P}\left(>\Delta_{1}\right)$ are found less than $5 \%$, suggesting a preferred alignment for the late-type S-mode SB galaxies (Table 3$)$. The auto correlation coefficient $(\mathrm{C} / \mathrm{C}(\sigma))$ and the hump at $>150^{\circ}$ support this result (Fig. 5f). The $\Delta_{11} / \sigma\left(\Delta_{11}\right)$ is found to be positive at $1.7 \sigma$ level, suggesting that the S-mode SBL galaxies tend to lie in the equatorial plane. Thus, the late-type S- and Z-mode SB galaxies show preferred and random alignments, respectively.

\subsubsection{Radial velocity}

The subsamples RV1 and RV2 show isotropy in all three statistical tests (Table 2). No humps or dips can be seen in Figs. 6a,b. Thus, the galaxies having radial velocity in the range 3000 $\mathrm{km} \mathrm{s}^{-1}$ to $4000 \mathrm{~km} \mathrm{~s}^{-1}$ show a random alignment for both the S- and Z-mode galaxies.

The humps at $90^{\circ}(>2 \sigma)$ and $110^{\circ}(>2 \sigma)$ are found in the Z- and S-mode RV3 galaxies, respectively (Fig. 6c). These two significant humps lead the subsample to show anisotropy in the statistical tests (Table 2). The $\Delta_{11}$ values are found negative at $\geq 1.5$ level, suggesting a similar preferred alignment for both modes: the galaxy rotation axes tend to be directed perpendicular to the equatorial plane.

A hump at $70^{\circ}(>1.5 \sigma)$ and a dip at $150^{\circ}(\sim 2 \sigma)$ cause the S-mode RV4 galaxies to show anisotropy in all three statistical tests (Fig. 6d). Thus, the S-mode galaxies having radial velocity in the range $4500 \mathrm{~km} \mathrm{~s}^{-1}$ to $5000 \mathrm{~km} \mathrm{~s}^{-1}$ show a similar preferred alignments as shown by the subsample RV3: galactic planes of galaxies tend to lie in the equatorial plane. The leading arm galaxies in the subsample RV4 show a random alignment (Table 3, Fig. 6d).

\subsubsection{Groups}

We do not study PA-distribution of S- and Z-mode galaxies in the groups Gr1, Gr3, Gr4 and Gr6 because of poor statistics (number $<50$ ). 

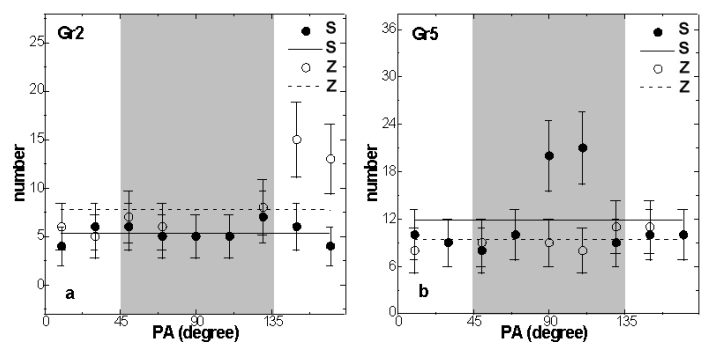

Fig. 7 The equatorial PA-distribution of Z- and S-mode galaxies in the groups Gr2 and Gr5. The abbreviations are listed in Table 1. The symbols, error bars, dashed lines and the explanations are analogous to Fig. 4.

We study the PA-distribution of S- and Z-mode galaxies in the groups Gr2 and Gr5, where the dominance of either Z- or S-mode is noticed. In addition, the statistics is relatively better in these groups.

In the group Gr2, Z-mode galaxies dominate the S-mode galaxies. In this group, the Z-mode galaxies show a preferred alignment whereas S-mode galaxies show a random alignment in the PA-distribution. All three statistical tests suggest anisotropy in the Z-mode galaxies (Table 3). The humps at $>150^{\circ}$ cause the $\Delta_{11}$ value to be positive at $>1.5 \sigma$ level (Fig. 7a), suggesting that the rotation axes of Z-mode galaxies in $\mathrm{Gr} 2$ tend to be oriented parallel the equatorial plane.

The S-mode galaxies dominate in the group Gr5. Interestingly, a preferred alignment of S-mode galaxies is noticed in the PA-distribution. In Fig. $7 \mathrm{~b}$, two significant humps at $90^{\circ}$ $(\sim 2 \sigma)$ and $110^{\circ}(>2 \sigma)$ can be seen. These humps lead the subsample (S-mode Gr5) to show anisotropy in the statistical tests (Table 3). No preferred alignment is noticed in the Z-mode galaxies of this group.

Thus, the dominating structural modes ( $\mathrm{Z}$ or $\mathrm{S}$ ) show a preferred alignment in the PAdistribution. This is noticeable result.

\subsection{Discussion}

Fig. 8 shows a comparison between the number $(\Delta)$ and position angle $\left(\Delta_{11} / \sigma\left(\Delta_{11}\right)\right)$ distribution of S- and Z-mode galaxies in the total sample and subsamples. This plot deals the correlation between the homogeneity in the structural modes and the random alignment in the subsamples. The grey-shaded region represents the region of isotropy and homogeneity for the $\Delta_{11} / \sigma\left(\Delta_{11}\right)$ and $\Delta(\%)$, respectively. Twenty five (out of $39,64 \%$ ) subsamples lie in the greyshaded region (Fig. 8a), suggesting a good agreement between the homogeneous distribution of S- and Z-mode galaxies and the random alignment of the rotation axes of galaxies. In four subsamples (SE, SBL, Gr2 and Gr5), a good correlation between the preferred alignment and the dominance of either S- or Z-mode galaxies is noticed (Fig. 8a). Thus, it is noticed that the random alignment of the PAs of galaxies hint the existence of inhomogeneity in the structural modes.

Aryal \& Saurer (2006) and Aryal, Paudel \& Saurer (2007) studied the spatial orientation of galaxies in 32 Abell clusters of BM type I, II, II-III and III and found a significant preferred alignment in the late-type cluster (BM type II-III, III). They concluded that the randomness decreases systematically in galaxy alignments from early-type (BM type I, II) to late-type (BM type II-III, III) clusters.

We noticed a very good correlation between the random alignments and the homogeneity in the structural modes. Probably, this result reveals the fact that the progressive loss of homo- 


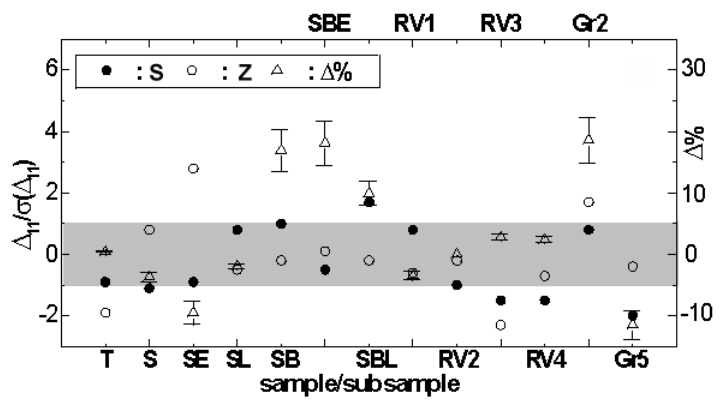

Fig. 8 A comparison between the number $(\Delta \%)$ and the position angle $\left(\Delta_{11} / \sigma\left(\Delta_{11}\right)\right)$ distribution of Z- and S-mode galaxies in the total sample and subsamples.

geneity in the structural modes might have some connection with the rotationally supported (spirals, barred spirals) to the randomized (lenticulars, ellipticals) system. Thus, we suspect that the dynamical processes in the cluster evolution (such as late-type clusters) give rise to a dynamical loss of homogeneity in the structural modes. It would be interesting to test this prediction by studying the S- and Z-type spirals in the late-type clusters in the future.

As $60 \%$ of galaxies in the nearby universe are rotationally supported discs, understanding angular-momentum acquisition is obviously a crucial part of understanding galaxy evolution. The winding sense of spiral arm patterns (morphological feature) allows us to infer the orientation of the angular-momentum vector of the disc galaxy. The expected distribution of spin vectors of galaxies shows markedly different trends according to the galaxy formation scenarios. One can suspect the possibility that the actual distribution of galaxy spin shows a dipole or a quadrupole component depending on the scenarios of galaxy formation. If galaxy spins were generated according to the primordial whirl scenario, a strong bias in either the $\mathrm{S}$ or $\mathrm{Z}$ patterns would be seen in a face-on sample of galaxies. We did not find this trend in our sample. A quadrupole distribution of $\mathrm{S} / \mathrm{Z}$ might be observed if the primary process was the generation of spins due to the pancake shock scenario or the explosion scenario. On the other hand, if the galaxy spins were produced by the tidal spin-up process, there would be no global anisotropy as we noticed in many cases, unless galaxy-cluster tidal interaction rather than galaxy-galaxy tidal interaction were the primary process. No significant correlation, however, was identified in any ensemble.

\section{CONCLUSIONS}

We studied the winding sense of 1621 field galaxies around the Local Supercluster. These galaxies have radial velocity (RV) in the range $3000 \mathrm{~km} \mathrm{~s}^{-1}$ to $5000 \mathrm{~km} \mathrm{~s}^{-1}$. The distribution of Z- and S-mode galaxies is studied in the total sample and 32 subsamples. To examine nonrandom effects, the equatorial position angle $(\mathrm{PA})$ distribution of galaxies in the total sample and subsamples are studied. In order to discriminate anisotropy from the isotropy we have performed three statistical tests: chi-square, auto-correlation and the Fourier. Our results are as follows:

1. The homogeneous distribution of the total Z- and S-mode galaxies is found, suggesting the homogeneous distribution of winding sense ( $\mathrm{S}$ or Z) of galaxies having RVs $3000 \mathrm{~km} \mathrm{~s}^{-1}$ to $5000 \mathrm{~km} \mathrm{~s}^{-1}$. The PA-distribution of S-mode galaxies is found to be random, whereas preferred alignment is noticed for Z-mode galaxies. It is found that the galactic rotation axes of Z-mode galaxies tend to be oriented perpendicular the equatorial plane. 
2. Z-mode are found $3.7 \%( \pm 1.8 \%)$ more than that of the S-mode in the spirals whereas a significant dominance $(17 \% \pm 8.5 \%)$ of S-mode is noticed in the barred spirals. This difference is found $>8 \%$ for the irregulars and the morphologically unidentified galaxies. A random alignment is noticed in the PA-distribution of Z- and S-mode spirals. Thus, it is noticed that the random alignment of the PAs of galaxies lead the existence of inhomogeneity in the structural modes of galaxies.

3. The inhomogeneity in the structural modes is found stronger for the late-type spirals (Sc, $\mathrm{Scd}, \mathrm{Sd}$ and $\mathrm{Sm}$ ) than that of early-type (Sa, Sab, Sb and Sbc). Similar result is found for the late-type barred spirals.

4. A very good correlation between the number of Z- and S-mode galaxies are found in the RV subsamples. All 4 subsamples show the $\Delta$ value less than $5 \%$. Thus, we conclude that the homogeneous distribution of structural modes of field galaxies remain invariant with the global expansion.

5. The galaxies having RVs $3000 \mathrm{~km} \mathrm{~s}^{-1}$ to $4000 \mathrm{~km} \mathrm{~s}^{-1}$ show a random alignment for both the Z- and S-modes. The rotation axes of Z- and S-mode galaxies having $4000<\mathrm{RV}(\mathrm{km}$ $\left.\mathrm{s}^{-1}\right) \leq 4500$ tend to be oriented perpendicular the equatorial plane.

6. The distribution of the winding sense of galaxies is found homogeneous in $\sim 80 \%$ area of the sky. This property is found to be violated in few groups of galaxies. Two such groups (Gr2 and Gr8) are identified. In these groups, the structural dominance and the preferred alignments of galaxies are found to oppose each other.

The true structural mode of a galaxy must involve a determination of which side of the galaxy is closer to the observer (Binney and Tremaine 1987). Three-dimensional determination of the leading and the trailing arm patterns in the galaxies is a very important problem. We intend to address this problem in the future.

Acknowledgements We are indebted to the referee for his/her constructive criticism and useful comments. I acknowledge Profs. R. Weinberger and W. Saurer of Innsbruck University, Austria for insightful discussions. I am thankful to Tribhuvan University, Nepal and Innsbruck University, Austria for providing financial assistance to visit Innsbruck University during JanMarch 2009.

\section{References}

Abell, G.O., Corwin, H.G., Olowin, R.P. 1989, ApJS, 70, 1

Aryal, B., Paudel, S., Saurer, W. 2007, MNRAS, 379, 1011

Aryal, B., Saurer, W. 2006, MNRAS, 366, 438

Aryal, B., Saurer, W. 2005, A\&A, 432, 841

Bergh, V.D. 1959, Publ. David Dunlop Obs. 2, 145

Bergh, V.D. 1966, AJ, 71, 922

Binney J., Tremaine, S. 1987, Galactic Dynamics, Princeton Univ. press, Princeton, New Jersey

Brandner, W., Grebel, E.K., Chu, Y.-H. et al. 2000, AJ, 119, 292

Brunzendorf, J., Meusinger H. 1999, A\&AS, 139, 141

Capozziello, S., Lattanzi, A. 2006, Ap\&SS, 301, 1-4, 189

Corwin, H.G., de Vaucouleurs, A., de Vaucouleurs, G. 1985, Univ. Texas Monogr. Astron., 4, 1

Dreyer, J.L.E. 1895, MNRAS, 51, 185

Dreyer, J.L.E. 1908, MNRAS, 59, 105

Doroshkevich, A.G. 1973, ApJ, 14, L11

de Vaucouleurs, G., de Vaucouleurs, A., Corwin, et al. 1991, Third Reference Catalogue of Bright

Galaxies, Springer-Verlag, New York

Godlowski, W. 1993, MNRAS, 265, 874

Impey, C.D., Sprayberry, D., Irwin, M.J., Bothun, G.D. 1996, Astrophys. J. Supp., 105, 209 
Kodaira, K., Okamura, S., Ichikawa, S. 1990, Photometric Atlas of Northern Bright Galaxies, Univ. of Tokyo Press, Tokyo

Lauberts, A. 1982, ESO/Uppsala Survey of the ESO B Atlas, Garching bei Muenchen

Longo, M. J. 2007, preprint (astro-ph/0703325)

Land K., Slosar A., Lintott C. et al. 2008, MNRAS, 388, 1686

MacAlpine, G.M., Smith, S.B., Lewis, D.W. 1977a, ApJS, 34, 95

MacAlpine, G.M., Smith, S.B., Lewis, D.W. 1977b, ApJS, 35, 197

MacAlpine, G.M., Lewis, D.W., Smith, S.B. 1977c, ApJS, 35, 203

MacAlpine, G.M., Lewis, D.W. 1978, ApJS, 36, 587

MacAlpine, G.M., Williams, G.A. 1981, ApJS, 45, 113

Markarian, B.E. 1967, Astrofizika, 3, 24

Markarian, B.E., Lipovetskii, V.A., Stepanian, D.A. 1983, Astrofizika, 19, 29

Messier, C. 1784, Catalogue des nebuleuses et des amas d'etoiles, Connaissance des Temps

Nilson, P. 1973, Uppsala General Catalogue of Galaxies, Nova Acta Uppsala University, Ser. V:A, Vol.1

Nilson, P. 1974, Upps. Astron. Obs. Rep., 5

Oort, J.H. 1970a, Science, 170, 1363

Oort, J.H. 1970b, A\&A, 7, 405

Ozernoy, L.M. 1978, in: Longair M.S., Einasto J., eds., The Large Scale Structure of the Universe, Proc.

IAU Symp. No. 79. Reidel, Dordrecht, p. 427

Peebles, P.J.E. 1969, ApJ, 155, 393

Pasha, I.I. 1985, Sov. Astron. Lett., 11, 1

Shectman, S.A., Landy, S.D., Oemler, A. et al. 1996, ApJ, 470, 172

Struble, M.F., Rodd, H.J. 1999, ApJS, 125, 355

Sugai, H. \& Iye, M. 1995, MNRAS, 276, 327

Takase, et al. 1980-2000, Kiso Ultraviolet Galaxy Catalog, Publ. Nat. Astron. Obs. Japan

Thomasson, M., Donner, K. J., Sundelius, B. et al. 1989, A\&A, 211, 25

Vorontsov-Vel, Y.B.A., Krasnogorskaya, A.A., Arkhipova, V.P. 1962-74, Morphological Catalogue of Galaxies (Part I-V), Trudy Gosud. Astron. Inst. Shternberga

Zwicky, F., Wild, P., Karpowicz, M., Kowal, C.T. 1961-68, The Catalogue of Galaxies and Clusters of Galaxies, California Inst. of Tech., Pasadena 